\title{
Behavioral appraisal by implementing a short sequence of stress resolves adaptively changed stress gains
}

\section{Eun-Hwa Lee}

Departments of Brain and Cognitive Sciences, Scranton College

Jin-Young Park

Ewha Womans University https://orcid.org/0000-0001-7277-0248

Hye-jin Kwon

Ewha Womans University

Pyung-Lim Han ( $\sim$ plhan@ewha.ac.kr)

Ewha Womans University

\section{Article}

Keywords: stress, glucocorticoids, depression,HPA axis, prelimbic cortex

Posted Date: October 20th, 2020

DOI: https://doi.org/10.21203/rs.3.rs-90131/v1

License: (c) This work is licensed under a Creative Commons Attribution 4.0 International License.

Read Full License

Version of Record: A version of this preprint was published at Nature Communications on November 18th, 2021. See the published version at https://doi.org/10.1038/s41467-021-26968-4. 


\section{Abstract}

Chronic stress produces adaptive changes in the brain via the cumulative action of glucocorticoids, which causes psychiatric illnesses such as depression. Here we show that a behavioral method implementing weak stress does not strengthen but resolves existing stress gains. Chronic stress produces persistent depressive behaviors in mice, and repeated daily treatment with 5-min restraint produces antidepressive effects. Repeated treatment with low-dose glucocorticoids mimics the anti-depressive effects of weak stress. Repeated weak stress or low-dose glucocorticoid treatment distinctively activates the prelimbic cortex (PL), and reverses the stress-induced altered gene expression profiles. Chemogenetic inhibition of $\mathrm{PL}$ outputs projecting to the nucleus accumbens, basolateral amygdala, or bed nucleus of the stria terminalis (BNST) dissipates antidepressive effects of weak stress, but only the PL-to-BNST circuit produces changes in dysregulated glucocorticoid release. Our results suggest that behavioral appraisal by implementing weak stress can resolve adaptively altered stress gains and rectify stress-induced depressive behaviors.

\section{Introduction}

Behavioral appraisals are used to treat the emotional dysfunction of psychiatric disorders ${ }^{1}$. Cognitive reappraisal is a cognitive method that regulates emotion by reinterpreting an emotion-provoking situation or reframing emotional expression ${ }^{2,3}$. A related but more complex form of behavioral appraisals includes cognitive behavioral therapy (CBT) $)^{4,5}$ and exposure therapy ${ }^{6}$, which involve stress responses. Although the behavioral appraisals are beneficial for post-traumatic stress disorder ${ }^{6,7}$ and depression ${ }^{4,8,9}$, the underlying neural mechanisms remain largely unknown, and it remains unclear if stress responses are required for therapeutic effects of the behavioral methods or they are an impediment to be properly controlled.

Chronic stress is a potent risk factor for various psychiatric illnesses, including depression ${ }^{10,11,12}$. Stress has been described as "the non-specific response of the body to any demand for change"13,14. The stress response proceeds by activating the hypothalamus-pituitary-adrenal (HPA) axis, which causes release of glucocorticoids (GCs, cortisol in humans and corticosterone in rodents) from the adrenal glands ${ }^{15,16}$. GCs normally stimulate energy metabolism and general physiological activity and increase vigilance, memory, and other cognitive functions ${ }^{17,18}$. Therefore, normal physiological stress has necessary and beneficial effects in daily life. Basal blood GC levels are low, but they exhibit circadian oscillation, with the highest levels in the early morning and the lowest levels at midnight. The daily fluctuation of GCs might be important for resetting physiological activities of the stress coping system, but the biological significance of low GC levels has not been carefully investigated.

Chronic stress produces changes in various brain regions beyond their homeostatic capability ${ }^{16,18,19}$. One of the brain regions that undergo functional and structural changes following chronic stress is the medial prefrontal cortex (mPFC) in rodents ${ }^{19,20,21}$. Chronic stress reduces glutamate transmission and related 
signaling events ${ }^{22,23}$, decreases neuronal activity ${ }^{23}$, and causes dendritic atrophy and spine loss in the $\mathrm{mPFC}^{24}$, which leads to persistent depressive behaviors. Furthermore, the reduced neuronal activity in the mPFC induced by stress leads to dysregulation of the HPA axis ${ }^{16,25}$. These results suggest that GCdependent changes in the MPFC are critical for stress-induced depressive behaviors. However, several studies have reported that glucocorticoid receptor (GR) agonists produce acute antidepressant effects. Dexamethasone treatment in conjunction with sertraline/fluoxetine for 4 days ${ }^{26}$ or a single intravenous injection of cortisol ${ }^{27,28}$ acutely improves depressive symptoms in depression patients. Low-dose corticosterone administered for 4 days in mice reduces immobility time in the forced swim test ${ }^{29}$. These results raise the possibility that GCs can exert anti-depressive effects under certain conditions. Thus, whether and if so how GC exerts pro-depressive or anti-depressive effects have been a long-standing unsolved problem ${ }^{30}$.

Chronic stress produces adaptive changes via the cumulative action of GCs. If existing stress gains are cumulatively strengthened by chronic stress, prior stress gains should be in an unstable state to integrate the incoming stress inputs. In the present study, we tested the hypothesis that behavioral method implementing weak stress could provide an opportunity to modify existing stress gains instead of strengthening them, and we demonstrated that a behavioral method implementing weak stress, but not strong stress, can rectify adaptively altered stress gains in the MPFC and rescue stress-induced depressive behaviors.

\section{Results}

\section{Strategic new stress changed adaptive stress gains}

We tested our hypothesis that a short sequence of behavioral stress would not strengthen existing stress gains but resolve them by applying brief restraint in stress-induced models of depression and assessing behavioral changes. Mice (C57BL/6) treated with daily 2-h restraint for 14 days (chronic restraint stress, CRST) showed reduced sociability in the two-chamber social interaction test (SIT), decreased sucrose preference in the two-bottle sucrose preference test (SPT), and increased immobility in the tail suspension test (TST) and forced swim test (FST) (Fig. 1a-e). We found that repeated stress induced by daily $\underline{5}$-min restraint for 14 days (RS5), which in itself did not produce depressive effects, reversed the stress-induced behavioral changes in CRST mice, as did the antidepressant imipramine (Fig. 1b-e). Interestingly however, treatment with daily 10-min or 15-min restraint for 14 days (RS10 and RS15, respectively) did not produce those changes (Fig. 1b-e). K-Means clustering, an unsupervised machine-learning algorithm that groups multiple factors into featured clusters, of the behavioral responses of all individuals, in conjunction with principal component analysis (PCA), yielded two clusters that contained the control or CRST groups in the SIT x SPT x [TST x FST] matrix (Fig. 1f). The RS5- and imipramine-treated individuals were distributed in the cluster containing most of the control animals, whereas the RS10- and RS15treated animals were in the cluster containing most of the CRST mice (Fig. 1f,g), suggesting that RS5 treatment completely reversed the CRST-induced changes at the individual level, not just in a group of 
animals. The anti-depressive effects of RS5 were stably maintained for one month (Fig. $1 \mathrm{~h}, \mathrm{i})$. Our doseranging study indicated that treatment with the 5-min restraint for 7 days or more, but not 3 or 5 days, produced anti-depressive effects (Extended Data Fig. 1a-f).

We also examined whether RS5 treatment was effective in the chronic social defeat stress (CSDS) model, which is unrelated to restraint stress (Fig. 1j). CSDS-susceptible mice exhibited behavioral deficits in the SIT, SPT, TST, and FST, and RS5 treatment of those mice restored the behavioral deficits in those tests (Fig. 1k-o), suggesting that RS5 effects do not result from a simple cognitive reinterpretation of the previous stress context.

Next, we examined whether RS5 could produce behavioral changes for ICR mice, an outbred line. The progenies of the normal $(\mathrm{N})$ and maternal stress (MS) groups of ICR mice were randomly allocated to receive CRST or CRST+RS5 (Extended Data Fig. 2a,b). CRST treatment in the $\mathrm{N}$ and MS groups produced decreased sociability in the SIT, reduced sucrose preference in the SPT, and increased immobility in the TST and FST. RS5 treatment of the CRST-treated N and MS groups reversed their stress-induced behavioral deficits (Extended Data Fig. 2c-f). Together, these results suggest that RS5 can modify adaptive behavioral changes induced by different chronic stressors.

\section{Low-dose glucocorticoids mimicked RS5 effects}

The serum corticosterone (CORT) levels induced by 5 -min or 15 -min restraint in naïve mice were much lower than those induced by 2-h restraint, but they cleared as slowly as the levels induced by 2-h restraint (Fig. 2a). The CORT level induced by a single 5-min restraint (S5) in CRST mice peaked at $124.2 \mathrm{ng} / \mathrm{ml}$ after $10 \mathrm{~min}$, which was higher than that $(97.4 \mathrm{ng} / \mathrm{ml})$ induced by S5 in naïve mice. Moreover, the CORT level induced by S5 in CRST mice cleared more slowly than that induced by S5 in naïve mice (Fig. 2b,c), but RS5 treatment in CRST mice returned the peak CORT level and its clearance time to those of the control (Fig. 2c,d). These results suggest that RS5 rectifies the stress-induced dysregulation of the HPA axis.

CRST mice had increased basal serum CORT levels compared with the naïve controls, and RS5 treatment in CRST mice restored their basal CORT levels to the controls (Fig. 2e). Interestingly, RS10 and RS15 treatment also significantly suppressed the basal CORT levels in CRST mice, although their effects were less dramatic than those of RS5 (Fig. 2e). CRST mice showed increased expression of corticotrophinreleasing hormone (CRH) and arginine vasopressin (AVP) in the paraventricular nucleus (PVN), and RS5 treatment in CRST mice suppressed the expression to the levels found in the naïve control. RS10 and RS15 treatment partially reduced CRH and AVP expression (Fig. 2f).

We tested whether post-stress treatment with low-dose CORT would mimic the effects of RS5. An intraperitoneal injection of CORT in naïve mice increased the serum CORT level in a dose-dependent manner. The serum CORT level following injection of $0.1 \mathrm{mg} / \mathrm{kg}$ of CORT was close to that induced by a 
5-min restraint (Fig. 2a,g). Repeated injection of CRST mice with $0.1 \mathrm{mg} / \mathrm{kg}$ of CORT, or even 0.5 or 1.0 $\mathrm{mg} / \mathrm{kg}$ of CORT, produced anti-depressive effects in the SIT, SPT, TST, and FST, and those behavioral changes were comparable to those induced by RS5 (Fig. 2h-l). Repeated injection of low-dose CORT in CRST mice also restored their increased basal CORT and reduced the increased weight of their adrenal glands to those of the naïve controls (Fig. 2m,n). PCA and $K$-Means clustering of behavioral and physiological factors in the [SIT x SPT] x [TST x FST] $x$ [adrenal gland weight] matrix indicated that behavioral recovery with CORT treatment proceeds with restoration of adrenal gland weight at the individual level (Fig. 2o,p).

\section{RS5 effects required activation of the HPA axis}

NBI27914 (an inhibitor of CRH receptor 1) or RU486 (an inhibitor of GR) treatment in CRST mice unexpectedly heightened the increased basal CORT level, but it produced a partial suppression of depressive-like behaviors in the SIT, SPT, TST, and FST. RS5 treatment while being treated with NBI27914 or RU486 in CRST mice did not fully suppress the increased basal CORT level and did not change the effects of NBI27914 or RU486 in the behavioral tests (Extended Data Fig. 3a-h).

Mice that received CRST treatment followed by adrenalectomy (ADX) surgery had a low basal CORT level and showed no CORT release after a 5-min restraint (Fig. 3a,b). RS5 treatment in mice with CRST plus ADX did not improve their stress induced behavioral deficits in the SIT, SPT, TST, and FST (Fig. 3c-h). $A D X$ alone in CRST mice did not change their depressive behavior (Fig. 3c-h). These results suggest that HPA axis activation is necessary for RS5 to produce therapeutic effects, whereas simple CORT depletion is ineffective.

\section{RS5 treatment activated core parts of the limbic system}

We investigated the brain regions recruited by RS5 using a stimulus-induced c-Fos mapping strategy. Repeated treatment with 5-min restraint in CRST mice increased c-Fos expression in the PL, ventral subiculum (vSub), dorsal bed nucleus of the stria terminalis (dBNST), PVN, and other parts of the limbic system (Fig.4a-c and Supplementary Table 1). Interestingly, the $c$-Fos levels induced by repeated treatment with 5-min restraint in those regions were higher than those induced by repeated treatment with 15 -min restraint or a single 5-min or 15-min restraint treatment. Among the $c$-Fos-positive neurons in the $\mathrm{PL}, 74.4 \%$ were glutamatergic, and $17.6 \%$ were GABAergic. Among the glutamate neurons, $46.8 \%$ were $c$ Fos-positive, whereas $44.2 \%$ of GABA neurons were c-Fos positive (Fig. $4 \mathrm{~d}-\mathrm{g}$ ).

Repeated injections of low-dose CORT $(0.1,0.5$ or $1.0 \mathrm{mg} / \mathrm{kg})$ in CRST mice increased c-Fos expression in the PL, BLA, NAc core (NAcc), vSub, dBNST, and PVN, the same regions recruited by repeated treatment with 5-min restraint (Extended Data Fig. 4a-c). 


\section{RS5 treatment restored altered expression of genes in the PL}

Next, we investigated whether RS5 affected genome-wide responses in the PL (Fig. 5a). Microarray analysis revealed that CRST treatment or RS5 in CRST-treated mice up- and downregulated 264 genes and 722 genes, respectively, by $\geq 1$.2-fold (Supplementary Tables 2 and 3). A heatmap presentation of the expression profiles of those genes that the CRST-induced altered gene expression profiles underwent a

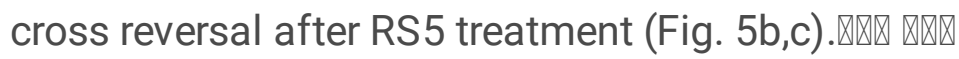

Gene Ontology (GO) enrichment analysis combined with K-Means clustering using the STRING database 22 showed that the genes identified above could be grouped into multiple clusters that presented various protein-protein interaction (PPI) networks. A serial $K$-Means clustering indicated that grouping those genes into 5-10 clusters $(k=5-10)$ built up PPI networks with functional modules relevant to the assumption of stress or glucocorticoid-related responses (Extended Data Fig. 5a). In the classification with 8 clusters, clusters 5 and 7, which contained 28 and 25 members, respectively, carried functional modules labeled with the GO terms "response to stress" or "response to glucocorticoid" (Fig. 5d,e). The remaining clusters are summarized in Extended Data Fig. 5b,c. Using the STRING database, the functional PPI networks formed by the clusters 5 and 7 were expanded by an additional 16 proteins that could participate as nodes (Fig. 5f).

\section{RS5 treatment upregulated GR and GluN subunits in PL neurons}

Chronic stress decreases GR expression and increases FK506-binding protein 5 (Fkbp5), a cellular factor that suppresses GR nuclear translocation ${ }^{32,33}$ in the mPFC ${ }^{34}$. Of the genes for stress or glucocorticoidrelated responses (Fig. 5f), GR expression in the PL was downregulated by CRST, whereas its expression was reversed to the control after RS5 or CORT $(0.1 \mathrm{mg} / \mathrm{kg})$ treatment. Fkbp5 and Fkbp4 were upregulated and downregulated, respectively, by CRST, whereas their altered expression was restored after RS 5 or CORT $(0.1 \mathrm{mg} / \mathrm{kg})$ treatment (Fig. $5 \mathrm{~g})$. Transcript levels of ERK1 and ERK2 declined after CRST, and their expression was reversed by CORT $(0.1 \mathrm{mg} / \mathrm{kg})$ treatment, but not by RS5. The transcript levels of mineralocorticoid receptor (MR), heat shock protein 90a, member A1 (Hsp90aa1), and dual specificity protein phosphatase 1 (Dusp1) tended to be changed by CRST, RS5, or CORT $(0.1 \mathrm{mg} / \mathrm{kg})$, although their changes overall were subtle (Fig. $5 g, h$ ).

Immunohistological analysis followed by $K$-Means clustering indicated that CRST increased Fkbp5 expression and decreased GR nuclear distribution at the level of single cells, whereas RS5 and CORT (0.1 $\mathrm{mg} / \mathrm{kg}$ ) treatment reverted their altered expression to the control. Interestingly, the positive correlation between Fkbp5 and GR expression was apparent in the CRST group, but not in the CON, RS5, or CORT groups (Fig. 5i-n). Small interfering RNA (siRNA)-mediated inhibition of GR increased Fkbp5 expression in the PL (Fig. 5o-q), increased CRH and AVP expression in the PVN (Fig. 5r), and induced depressive 
behaviors in the TST and FST (Fig. $5 \mathrm{~s}, \mathrm{t}$ ). These results suggest that GR expression declined in PL neurons after CRST, and Fkbp5 levels increased, whereas RS5 and low-dose CORT treatment restored the altered GR and Fkbp5 expression.

Repeated stress suppresses the expression of the GluA and GluN subunits in the prefrontal cortex ${ }^{23,35}$. Consistently, CRST treatment reduced the expression of the GluN subunits NR1, NR2A, and NR2B in the $\mathrm{PL}$, whereas repeated weak stress or repeated treatment with CORT $(0.1 \mathrm{mg} / \mathrm{kg})$ restored their reduced expression to the control level (Extended Data Fig. 6a-h).

\section{RS5 treatment rectified the increased expression of CaMKIla in the PL}

Western blot analyses indicated that p-CaMKIla and p-ERK1/2 levels in the PL were upregulated and downregulated, respectively, after CRST, and that the altered expression of p-CaMKIla, but not p-ERK1/2, was restored to the control after RS5 treatment (Fig. 6a-c). p-CaMKIla was expressed mostly in glutamatergic neurons (Fig. 6d,e). Immunohistological analysis followed by $K$-Means clustering indicated that CRST increased p-CaMKIla expression in PL neurons while decreasing GR expression, and that their altered expression was restored to the control after RS5 or CORT $(0.1 \mathrm{mg} / \mathrm{kg})$ treatment. p-CaMKIlla and GR expression levels were positively correlated at the single-cell level throughout CRST, RS5 or CORT ( 0.1 $\mathrm{mg} / \mathrm{kg}$ ) treatment (Fig. $6 \mathrm{f}-\mathrm{k}$ ). siRNA-mediated inhibition of CaMKlla increased GR expression in the PL, whereas inhibition of ERK1 or ERK2 did not. Fkbp5 expression was partially affected by the inhibition of ERK2 (Fig. 6I-p). In fact, siRNA-mediated inhibition of CaMKIla but not ERK1 and ERK2, in the PL of CRST-treated mice restored the reduced sociability and increased immobility time in the TST and FST (Fig. 6q-s).

CRST decreased the expression of the GABAA receptor subunits GABRa1 and GABRß2 in the PL, and RS5 or CORT $(0.1 \mathrm{mg} / \mathrm{kg}$ ) treatment increased their reduced expression (Fig. 7a). In mice exposed to CRST followed by RS5, inhibition of GABAA receptors in the PL by infusion of picrotoxin through a preimplanted cannula upregulated p-CaMKIlla levels and promoted depressive-like behavior in the TST and FST (Fig. 7b-g). These results suggest that p-CaMKIla upregulation in the PL can be induced by a decrease in GABAA receptor function.

Interestingly, local inhibition of CaMKIla in the PL by stereotaxic infusion of siRNA-CaMKIla or KN62 (an inhibitor of CaMKIla) in normal mice produced depressive-like behaviors in the SIT, TST, and FST, suggesting that reduced levels of CaMKIla in the PL are also pro-depressive (Fig. $7 \mathrm{~h}-\mathrm{m}, \mathrm{p}-\mathrm{s}$ ). In contrast, siRNA-mediated or U0126-mediated inhibition of ERK1 in the PL in normal mice reduced immobility time in the TST and FST, although it produced no effect in the SIT (Fig. 7h-m). p-ERK1/2 was expressed mostly in glutamatergic neurons (Fig. 7n,o). These results suggest that CRST upregulates $\mathrm{p}$-CaMKIla in PL neurons, which promotes depressive behavior, whereas RS5 produces anti-depressive effects by downregulating p-CaMKIla in PL neurons. 


\section{Activation of PL neurons was required for RS5 effects}

Given that RS5 upregulated GR, GluN, and GABAA receptor subunits in PL neurons (Figs. $5 \mathrm{~g}$ and $7 \mathrm{a}$ and Extended Data Fig. 6a) and that the PL had distinct c-Fos expression following repeated 5-min restraint (Fig. $4 a-c)$, we investigated whether the neural activity of PL neurons was required for RS5 to have antidepressive effects. Mice injected in the PL with the AAV8-CaMKIla-hM3D(Gq) or AAV8-hSynhM3D $(\mathrm{Gq})$ vector were subjected to CRST. Clozapine N-oxide (CNO) injection in CRST mice carrying an excitatory vector increased c-Fos expression in PL neurons (Extended Data Fig. 7a-f). CNO treatment in mice with $\mathrm{hM} 3 \mathrm{D}(\mathrm{Gq})$ expression driven by the CaMKIla promoter, but not the human synapsin (hSyn) promoter, produced increased sociability in the SIT and decreased immobility time in the TST and FST (Extended Data Fig. 7g-k). After 7 days of CNO washout, mice with CaMKIla-hM3D(Gq) showed a relapse of depressive behaviors and had a substantial increase in their basal CORT level relative to the control (Extended Data Fig. 7l-q). These results suggest that activation of PL glutamatergic neurons can suppress depressive phenotypes in CRST-treated mice, but those rescue effects are transient.

Mice injected in the PL with the AAV8-CaMKIla-hM4D(Gi) inhibitory vector were subjected to CRST and then treated with RS5 or RS5+CNO. CNO injection in those mice partially blocked the RS5-induced suppression of the basal CORT level and dissipated the antidepressive effects of RS5 in the SIT, SPT, TST, and FST (Fig. 8a-k). These results suggest that RS5 produces anti-depressive effects by activating PL glutamatergic neurons.

\section{Multiple PL outputs mediated the effects of RS5}

The PL sends collaterals to the $\mathrm{ABNST}, \mathrm{BLA}$, and NAcc, the brain regions recruited by repeated treatment with 5-min restraint (Fig. 4a,b). We investigated whether the effects of RS5 require multiple PL output pathways or a specific efferent circuit.

The dBNST, which receives glutamatergic inputs from the PL (Extended Data Fig. 8a-d and 8h-k) and vSub (Extended Data Fig. 8I), contains GABA neurons that project to the PVN ${ }^{36,37}$. To test whether the PLàdBNST circuit mediated the effects of RS5, the PLàdBNST circuit was labeled with the AAV-DIO$\mathrm{hM} 4 \mathrm{D}(\mathrm{Gi})$-mCherry inhibitory vector using a retrograde Cre vector (Fig. 9a-c). CNO-mediated inhibition of the PLàdBNST circuit suppressed RS5-induced c-Fos expression in dBNST neurons, which were GAD67positive. However, that inhibition did not change RS5-induced c-Fos expression in the PVN (Fig. 9d-f). CNO-mediated inhibition of the PLàdBNST circuit in CRST mice dissipated the antidepressive effects of RS5 in the SIT, SPT, TST, and FST, and the normalization of the basal CORT level by RS5 (Fig. 9g-m). These results suggest that activation of the PLàdBNST is required for RS5 to have antidepressive effects and restore defective basal CORT release. 
Next, we examined whether PLàBLA and PLàNAcc neurons played a role in regulating the effects of RS5. The BLA and NAcc receive glutamatergic inputs from the PL (Extended Data Fig. 8e,f). The PLàBLA and PLàNAcc circuits were specifically labeled with the AAV-DIO-hM4D(Gi)-mCherry inhibitory vector and the retrograde Cre vector (Fig. 9n,o). CNO-mediated suppression of the PLàBLA or PLàNAcc circuit decreased RS5-induced c-Fos expression in the BLA and NAcc, respectively (Fig. 9p-s). Inhibiting the PLàBLA or PLàNAcc circuit in CRST mice with CNO during RS5 treatment blocked the anti-depressive effects of RS5 in the SIT, SPT, TST, and FST, whereas inhibition of those circuits did not change the RS5-induced normalization of basal CORT levels (Fig. 9t-x). These results indicate that activation of the PLàBLA and PLàNAcc circuits facilitates the anti-depressive effects of RS5, but the activation of those pathways is not essential for the recovery of the altered basal CORT level.

\section{Discussion}

The finding that behavioral method implementing a short sequence of stress arousals and its resolutions can rectify persistent behavioral changes in multiple models of depression highlights the feasibility of fighting adaptively altered stress gains with behavioral stress. Chronic restraint produces depressive behaviors that last for more than 3 months ${ }^{38,39}$. Despite those adaptive changes, RS5, but not RS10 or RS15, rectified depressive behaviors as did the antidepressant imipramine (Fig. 1). RS5 treatment did not produce anti-depressive effects when the HPA axis was blocked (Fig. 3 and Extended Data Fig. 3). Repeated injection with low-dose GC $(0.1 \mathrm{mg} / \mathrm{kg}$ ) recapitulated the effects of RS5 (Fig. $2 \mathrm{~h}-\mathrm{I})$. Paradoxically, however, repeated injection with GC at a dose higher than that induced by 10 -min or 15 -min restraint, and repeated injection with even $1.0 \mathrm{mg} / \mathrm{kg}$ of GC, which was comparable to the GC level induced by 2-h restraint (Fig. 2a,g), also produced anti-depressive effects (Fig. $2 \mathrm{~h}-\mathrm{I}$ ). These results suggest that behavioral appraisal by implementing a short sequence of stress produces anti-depressive effects via a GC-dependent mechanism. However behavioral method has a limited window to produce anti-depressive effects.

Chronic stress changes various brain regions beyond the homeostatic range by activating the HPA axis $^{16,18,19}$. Patients with depression have increased basal serum GC levels ${ }^{40,41}$. In contrast, RU486 (mifepristone), a GR antagonist, is beneficial for patients with psychotic depression ${ }^{42}$ and bipolar disorder ${ }^{14}$. Mice exposed to chronic stress have increased basal serum GC levels (Fig. 2). Administration of high-dose GC in drinking water ( $35 \mu \mathrm{g} / \mathrm{ml} /$ day) for 4 weeks $^{43}$ or subcutaneous injection of GC at a dose of 10,20 or $40 \mathrm{mg} / \mathrm{kg} /$ day for 21 days ${ }^{44}$ in rats mirrors the stress-induced dysfunction of the HPA axis and produces depressive-like behaviors. Therefore, GC is regarded as a mediator of chronic stress $^{15,16,17}$.

In the present study, we demonstrated that repeated treatment with a short sequence of behavioral stress or repeated injection with GC $(0.1-1.0 \mathrm{mg} / \mathrm{kg} /$ day) produced anti-depressive effects (Figs. 1 and $2 \mathrm{~h}-\mathrm{l})$ and reversed stress-induced molecular changes (Fig. 5) as did imipramine. The key findings are summarized in Fig. 10. These results raise the following important and related points. First, the fact that 
GC induction by behavioral stress and exogenous GC resolved existing stress gains suggests that GC functions as a stress modifier, which contradicts the classical conception that GC is a stress mediator. Although when and how GC functions as a stress modifier need to be studied in more detail, we speculate that repeated weak stress can restore stress coping ability, presumably by repeatedly boosting the feedback and feedforward regulatory mechanisms of the HPA axis (Fig. 10b,c,e). This possibility does not conflict with the classical conception that chronically imposed GC produces cumulative effects on stress gains due to the points described below. It will be worth studying whether the circadian oscillation of basal GC levels ${ }^{45,46}$, which is disrupted in patients with depression ${ }^{40,41}$, functions as a stress modifier by performing a daily reset of the stress coping system. In healthy individuals, the basal serum GC levels vary throughout the day, with the highest in the early morning and then falling throughout the day to the late evening. Second, repeated treatment with the behavioral stress or repeated treatment with GC could be used as an antidepressant strategy. Although the behavioral method has a narrow window to afford therapeutic effects, it could have an advantage over the pharmacological method. On the other hand, challenging with GC provides a more wider and more versatile therapeutic window to resolve existing stress gains. The profound therapeutic effects of behavioral stress and exogenous GC demonstrated in this study warrant further investigation. Third, the finding that repeated weak stress or treatment with even high-dose GC did not strengthen existing stress gains but resolved them (Figs. 1 and 2), raises the possibility that prior stress gains might become transiently deconsolidated and labile upon new stress inputs or GC flux. As stress-induced adaptive changes are deconsolidated by GC, brain cells appear to restore their normal homeostatic stability and physiological function to produce normal behavioral outputs. It will be worth studying the key factors and underlying signaling networks that regulate GCdependent changes and the mechanisms of homeostatic restoration.

Chronic stress increased the p-CaMKII level, which downregulated GR expression in PL neurons (Fig. $6 a, p)$. Local inhibition of CaMKIla in the PL produced anti-depressive effects in CRST-treated mice (Fig. $6 \mathrm{I}-\mathrm{s}$ ), and siRNA-mediated inhibition of GR in the PL of normal mice increased Fkbp5 and produced depressive-like behaviors (Fig. 7o-t). p-CaMKIla upregulation in PL neurons appears to be caused by stress-induced downregulation of GABAA receptors (Fig. 7). Fkbp5, in coordination with Hsp90, negatively regulates GR nuclear translocation ${ }^{33,47}$, so a stress-induced increase of $p$-CaMKII in the PL facilitates a vicious cycle in the GR $\downarrow$ à Fkbp5 $\uparrow$ pathway. These results suggest that stress-induced upregulation of pCaMKIla in the PL is a critical player mediating the effects of chronic stress on depressive behaviors.

Our results indicate that PL neurons and their associated neural systems, including the NAc, BLA, and BNST, compose the critical neuronal nodes and edges that support stress-induced depression and the modification of stress-induced changes by strategic weak stress, as summarized (Fig. 10b-e).

Glutamate/glutamine levels and the neural activity of glutamatergic neurons in the MPFC are reduced in CUMS, CRST, and CSDS-induced depression models in mice ${ }^{48,49,50}$. Chemogenetic activation of PL neurons facilitated anti-depressive effects and suppressed the stress-induced increase in basal GC levels, whereas chemogenetic inhibition of PL neurons during RS5 dissipated its anti-depressive effects (Figs. 8 and Extended Data Fig. 7). Furthermore, chemogenetic inhibition of the PLà NAc, PLà BLA or PLà dBNST

Page $10 / 49$ 
circuits during RS5 blocked its anti-depressive effects (Fig. 9), suggesting that activation of those circuits is required for RS5 to produce anti-depressive effects. Previous studies reported that activation of the $\mathrm{PL}^{51}$, the PLà NAc circuit ${ }^{52}$ or the PLà BLA circuit ${ }^{53}$ produced anti-depressive effects, which is partly consistent with our results. Interestingly, however, the PLà dBNST circuit played a role in the recovery of basal GC levels by RS5, whereas the PLà NAc and PLà BLA circuits did not (Fig. 9), raising the question if the latter cases, which did not recover normal HPA function, produce stable recovery from stress-induced changes. Overall, our results suggest that although the PL is the key area recruited by RS5, the PLà NAc, PLà BLA, and PLàdBNST pathways comprise the critical neural nodes and circuits that support the behavioral effects of RS5, and the PLàdBNST circuit supports the recovery of the HPA axis by RS5 (Fig. 10b-e).

Stress inoculation is a pretreatment strategy that improves subsequent stress coping and emotional regulation ${ }^{45}$. Stress inoculation in mice, by placing them behind a mesh-screen barrier in a cage containing an aggressor mouse for $15 \mathrm{~min}$, enhances subsequent stress coping behavior and cognitive function ${ }^{55}$. Another form of stress inoculation, called predictable chronic mild stress (PCMS), uses 5-min restraint for 28 days to improve mood, hippocampal neurogenesis and memory in rats ${ }^{56}$. PCMS treatment of rats in their early adolescence increases resilience to chronic unpredictable mild stress in adulthood ${ }^{57}$. In those studies, stress inoculation or PCMS is regarded as an immunization to enhance coping ability for future stress ${ }^{54}$. PCMS treated for 28 days induced anti-depressive effects in rats ${ }^{56}$. In contrast, our RS5 treatment (daily 5-min restraint for 14 days) in normal mice did not induce depressive behaviors (Fig. 1a-g), Therefore, it will be worth studying that a pretreatment paradigm of daily 5-min restraint for 7-14 days in normal mice would also produce resiliency to future chronic stress. It is possible that stress inoculation/PCMS and RS5 could commonly have a certain neural mechanism. Nonetheless, our experimental procedure provoking weak stress deals with a therapeutic strategy, whereas stress inoculation/PCMS is preventative.

Fig. 1

\section{Fig. 1 Repeated treatment with a short sequence of behavioral stress produces anti-depressive effects in stress-induced models of depression.}

a, Experimental design. Mice were treated with 5-min or 2-h restraint for 14 days. CRST mice were treated with 5-min, 10-min, or 15-min restraint or imipramine for 14 days (RS5, RS10, RS15, and IMI, respectively), and then given behavioral tests.

b- g, Representative tracks and \% time spent in the target and non-target chambers in the SIT (b), sucrose preference in the SPT (c), and immobility time in the TST (d) and FST (e) for the indicated groups on post-stress days 15-17. $K$-Means clustering $(k=2)$ of individuals in the SIT x SPT $x$ [TST x FST] matrix ( $f$ ) and \% composition of each group in the clusters $(\mathbf{g})$. PCA was used for dimension reduction of the TST $x$ FST components (PVE, 81.3\%) ( $n=9-10$ per group). 
h-l, Immobility time in the TST (h) and FST (i) for the indicated groups on post-stress days 43 and 44 ( $n$ = 8-10 per group). j, Experimental design. The susceptible mice were treated with RS5, and then given behavioral tests.

$\mathbf{k}-\mathbf{0}$, Mice susceptible or resilient to CSDS were separated by the sociability ratio (k). \% time spent in the target chamber in the two-chamber SIT (I), sucrose preference in the SPT (m), and immobility time in the TST (n) and FST (o) for the indicated groups ( $n=8-11$ per group).

Data are mean \pm SEM. * , difference compared to control; \#, difference compared to CRST. *, \#, $p<0.05$; $* \star$, $\# \#, p<0.01$ (One-way ANOVA followed by Newman-Keuls post-hoc test). See Supplementary Table 4 for statistical details.

Fig. 2

Fig. 2 RS5 and low-dose CORT normalize stress-induced dysregulation of the HPA axis.

a, Serum CORT levels in mice treated with 5-min, 15-min, 60-min, and 120-min restraint ( $n=8-9$ per group).

b-f, Experimental design (b). Time course of serum CORT levels in the indicated groups after exposure to a single 5-min restraint (S5) (Exp \#1) (c), and the area under the curve (AUC) between -5 min and 120 min (d) ( $n=7-8$ per group). Basal serum CORT levels (e) and CRH and AVP expression levels in the PVN (f) of the indicated groups (Exp \#2) ( $n=7-8$ per group).

g, Changes in serum CORT levels in mice after CORT injection ( $n=8-9$ per group).

h-p, Experimental design (h). CORT was injected for 14 days $(0.1,0.5,1.0 \mathrm{mg} / \mathrm{kg} / \mathrm{day}$, i.p.) in CRST mice. Behavioral performance in the SIT (i), SPT (j), TST (k), and FST (I) for the indicated groups ( $n=10$ per group). Basal serum CORT levels ( $n=8$ per group) (m) and adrenal gland (AG) weight ( $n=10$ per group) (n) of the indicated groups on post-stress day 22. $K$-Means clustering of individuals in the [AG weight] $x$ [SIT x SPT] x [TST x FST] matrix (0) and \% composition of each group in the clusters (p). PCA was used for dimensional reduction of the SIT and SPT components (PVE; 76.0\%) and the TST and FST components (PVE; 73.4\%).

Data are mean \pm SEM. *, difference compared to control; \#, difference compared to CRST. *, \#, $p<0.05$; $* *$, $\# \#, p<0.01$ (One-way ANOVA followed by Newman-Keuls post-hoc test). See Supplementary Table 4 for statistical details.

Fig. 3

Fig. 3 Adrenalectomy (ADX) blocks the antidepressive effects of RS5 in CRST mice. 
a, Experimental design for treatment with CRST, ADX or sham surgery, followed by RS 5 treatment and behavioral tests. Red arrows $(\downarrow)$ in Exp \#1, Serum collection point. Mice were sacrificed after exposure to a single 5-min restraint (S5).

b-h, The basal CORT levels at $-5 \mathrm{~min}$, and the time course of serum CORT levels in the indicated groups after exposure to S5 (Exp \#1) (b) ( $n=7-10$ per group). Social interaction in the SIT (c), sucrose preference in the SPT (d), and immobility time in the TST (e) and FST (f) for the indicated groups (Exp \#2). K-Means clustering of individuals in the SIT x SPT x [TST x FST] matrix ( $(\mathbf{g})$ and \% composition of each group in the clusters (h). The TST and FST components were transformed into linear eigenvectors using PCA (PVE; $78.2 \%)(n=7-10$ per group).

Data are mean \pm SEM. *, difference compared to control; \#, difference compared to CRST; + , difference compared to CRST+RS5. * \#, +, $p<0.05 ; * \star, \# \#,++, p<0.01$ (One-way ANOVA followed by Newman-Keuls post-hoc test). See Supplementary Table 4 for statistical details.

Fig. 4

\section{Fig. 4 Repeated treatment with a short sequence of behavioral stress recruits the brain regions regulating stress coping in CRST mice.}

a, Experimental design. CRST mice were treated with a single 5-min or 15-min restraint (S5 and S15, respectively) on post-stress day 8 , and then sacrificed, 20 min and 10 min later, respectively. Another group of CRST mice was treated with 5-min or 15-min restraint for 8 days (S5x8d and S15x8d, respectively), and then sacrificed, $20 \mathrm{~min}$ and $10 \mathrm{~min}$, respectively, after the last restraint.

b,c, Quantification of $c$-Fos expression levels in the PL, BLA, NAcc, vSub, dBNST, ventral BNST (vBNST), and PVN (b). Photomicrographs showing c-Fos expression in the PL (red box) (C) of the S5, S15, S5x8d, and S15x8d groups ( $n=4-6$ per group).

d-g, Photomicrographs showing c-Fos induction in GLU-4-positive or GAD67-positive neurons in the PL (d), and their quantification levels (e- $\mathbf{g})$ ( $n=6$ animals for each).

The details are shown in Supplementary Table 1. Data are mean \pm SEM. *, **, difference compared to control; \#, difference compared to CRST; +, difference between indicated groups. ${ }^{*}, \# \#,++, p<0.01$ (oneway ANOVA followed by Newman-Keuls post-hoc test). See Supplementary Table 4 for statistical details.

Fig. 5

Fig. 5 RS5 treatment reverses stress-induced changes in gene expression profiles in the PL.

a-c, Experimental design (a). Heatmap showing the expression profiles were changed by $\geq 1.2$-fold after CRST (b) and reversed by RS5 treatment in CRST mice (c), and follow-up unbiased alignment with the 
expression levels of the respective genes changed by RS5 (b) and CRST (c). $\uparrow$ and $\downarrow$, indicate up- and down-regulation, respectively.

d-f, GO enrichment analysis with weighted $K$-Means clustering identified the clusters which carry the functional modules labeled with the GO terms "response to stress" or "response to glucocorticoid" (d, e). The PPI networks formed by the members of clusters 5 (orange, 28 genes) and 7 (green, 24 genes) and 16 genes (black) from an extended search of the STRING database at a confidence level of $>0.700$ (f).

g,h, Expression levels of Nr3c1, Nr3c2, Fkbp5, Fkbp4, Hsp90aa1, and Hsp90ab1 (g), and Dusp1, CaMKIla, Mapk3, and Mapk1 (h) in the PL of the indicated groups ( $n=8-12$ per group).

i-n, Immunofluorescence images showing GR (red) and Fkbp5 (green) expression in the PL from the indicated groups (i). DAPI, blue. Quantification levels of total GR/DAPI (j), Fkbp5/DAPI (k), and DAPI intensity (I) in individual cells ( $n=4-6$ animals per group; $n=248-309$ per group). Differential expression of nuclear GR and Fkbp5 in individual cells $(\mathbf{m})$. Two distinct clusters with centroids classified by $K$-Means clustering, and the regression values $(\mathbf{m})$ and \% composition of individuals cells of each group ( $\mathbf{n})$ in the clusters were indicated.

o-t, Experimental design (o). siRNA-mediated knockdown of GR in the PL, and subsequent behavioral tests. Red arrow, time point for tissue prep. Transcript levels of GR (p) and Fkbp5 (q) in the PL and of CRH and AVP (r) in the PVN. Immobility time in the TST (s) and FST (t) for each group.

Data are mean \pm SEM. *, difference compared to control; \#, difference compared to CRST. *, \#, $p<0.05$; **, $\# \#, p<0.01$ (One-way ANOVA followed by Newman-Keuls post-hoc test). See Supplementary Table 4 for statistical details.

Fig. 6

Fig. 6 RS5 restores increased p-CaMKlla expression in PL neurons.

a-c, Western blots showing expression levels of p-CaMKIla, CaMKIlla, p-ERK1/2, ERK1/2, and $\beta$-actin in the PL of the indicated groups (a). Quantification levels of p-CaMKIla (b) and p-ERK1/2 expression (c) ( $n=6$ animals per group, 4 repeats).

d,e, Immunofluorescence staining of p-CaMKIlla (green) in PL neurons stained with GLU-4 or GAD67 (red) (d). Quantification levels (e) ( $n=4$ per group).

$\mathbf{f}-\mathbf{k}$, Immunofluorescence staining of p-CaMKIla (red) and total GR (green) in PL neurons (f). DAPI, blue. Differential expression of $\mathrm{p}$-CaMKIla and total GR in individual cells of the indicated groups (g). Two distinct clusters with centroids and regression values $(\mathbf{g})$ and the \% composition of individuals cells of each group in the clusters (h). Quantification levels of p-CaMKIla (i), GR (j) and DAPI (k) intensity in individual cells ( $n=4-6$ animals per group; $n=345-390$ per group). 
I-s, Experimental design (I). siRNA-mediated knockdown of CaMKIlla (m), ERK1 (n), and ERK2 (o) in the PL of CRST-treated mice. Red arrow, time point for tissue prep. Transcript levels of GR and Fkbp5 (p) in the PL of the indicated groups. Behavioral performance in the SIT (q), TST (r), and FST (s) for the indicated groups.

Data are mean \pm SEM. ${ }^{*}$, difference compared to control; \#, difference compared to CRST. * $\#, p<0.05, * *$, $\# \#, p<0.01$ (Student's t-test; One-way ANOVA followed by Newman-Keuls post-hoc test). See Supplementary Table 4 for statistical details.

\section{Fig. 7}

\section{Fig. 7 CaMKIla in the PL is a critical player regulating depressive behaviors.}

a, Transcript levels of GABRa1 and GABRB2 in the PL of the indicated groups. Sample groups were prepared from the experiments depicted in Fig. $4 \mathrm{~g}, \mathrm{~h}$ ( $n=7-12$ per group).

b-g, Experimental design (b). The picrotoxin (PTX) was infused using a pre-implanted cannula into the PL of mice subjected to CRST and RS5. Behavior tests were carried out between 60 and 90 min after drug infusion on post-stress day 22 (b). Western blots showing the expression levels of $p$-CaMKIla, CaMKIla, $p$ ERK1/2, ERK1/2, and $\beta$-actin in the PL of the indicated groups (c). Quantification levels of $p$-CaMKIla (d) and p-ERK1/2 (e) ( $n=6$ mice per group, 3 repeats). Behavioral performance in the TST (f) and FST (g) for the indicated groups ( $n=8$ per group).

$\mathbf{h}-\mathbf{m}$, Experimental design (h). siRNA-mediated knockdown of CaMKIla (i) and ERK1 (j) in the PL. Behavioral tests were carried out 2 days later in the order SIT, TST, and FST. Red arrow, time point for tissue prep. Behavioral performance in the SIT (k), TST (I), and FST $(\mathbf{m})$ for the indicated groups $(n=8$ per group).

$\mathrm{n}, \mathbf{0}$, Immunofluorescence staining of p-ERK1/2 (green) expression in the PL stained with GLU-4 or GAD67 (red) (n). Quantification levels of colocalization (o) ( $n=4$ per group).

p-s, Experimental design (p). KN-62 (CaMKlla inhibitor, $2.5 \mathrm{nmol} /$ injection), or Veh was infused into the PL through a pre-implanted cannula. After $60 \mathrm{~min}$, behavioral tests were carried out in the order SIT, TST and FST. Behavioral performance in the SIT (q), TST (r), and FST (s) for the indicated groups $(n=7$ per group).

Data are mean \pm SEM. *, difference compared to control; \#, difference compared to CRST. *, \#, $p<0.05$, **, $\# \#, p<0.01$ (Student's t-test; One-way ANOVA followed by Newman-Keuls post-hoc test). See Supplementary Table 4 for statistical details.

Fig. 8 
Fig. 8 Chemogenetic inhibition of PL neurons blocks the anti-depressive effects of RS5.

a, Experimental design. Mice injected in the PL with AAV8-CaMKIIa-hM4D(Gi)-mCherry inhibitory vector were subjected to CRST, followed by RS5 treatment with Veh or CNO injection.

b-e, Injection of the AAV8 vector and resulting mCherry expression in the PL (b). S5-induced c-Fos expression levels in the PL after CNO treatment (Exp \#1) (c and d) ( $n=4-6$ per group). Basal CORT levels in the indicated groups on post-stress day 22 (e) ( $n=7-8$ per group).

$\mathbf{f}-\mathbf{k}$, Behavioral performance in the SIT (f), SPT (g), TST (h), and FST (i) for the indicated groups (Exp \#2). K-Means clustering of individuals in the SIT $x$ SPT $x$ [TST x FST] matrix (J) and \% composition of each group in the clusters (K) ( $n=7-9$ per group).

Data are mean \pm SEM. *, difference compared to control; \#, difference compared to CRST. *, \#, $p<0.05$, **, $\# \#, p<0.01$ (Student's t-test; one-way ANOVA followed by Newman-Keuls post-hoc test). See Supplementary Table 4 for statistical details.

Fig. 9

Fig. 9 Chemogenetic inhibition of PL neurons projecting to the dBNST, BLA or NAcc blocks the antidepressive effects of RS5.

a, Experimental design. Mice injected in the PL with the AAV8-hSyn-hM4D(Gi)-mCherry and retrograde Cre vector in the dBNST, BLA, or NAcc were subjected to CRST, followed by RS5 treatment with Veh or CNO injection.

b,c, Injection of the AAV8 vector and resulting mCherry expression in the PL (b) and the PLàdBNST circuit (c), which was labeled with $\mathrm{hM} 4 \mathrm{D}(\mathrm{Gi})$ by injection of viral vectors $(\mathbf{a})$.

$\mathbf{d}-\mathbf{f}$, S5-induced c-Fos expression levels in the dBNST (d and $\mathbf{e}$ ) and PVN (f) after CNO treatment (Exp \#1) ( $n=4$ per group). c-Fos, red; GAD67, green; DAPI, blue.

g, Basal CORT levels in the indicated groups on post-stress day 22 ( $n=8$ per group).

$\mathbf{h}-\mathbf{m}$, Behavioral performance in the SIT (h), SPT (i), TST (j), and FST (k) for the indicated groups (Exp \#2). K-Means clustering of individuals in the SIT x SPT x [TST x FST] matrix (I) and \% composition of each group in the clusters $(\mathbf{m})$ ( $n=8$ per group).

$\mathbf{n}, \mathbf{0}$, The PLàBLA (n) or PLàNAcc (o) circuits, which were labeled with hM4D(Gi) by injection of viral vectors (a).

$\mathbf{p}-\mathbf{s}$, S5-induced $c$-Fos expression levels in the BLA ( $n=3-4$ per group) ( $\mathbf{p}$ and $\mathbf{q})$ and NAcc $(n=3-4$ per group) ( $\mathbf{r}$ and $\mathbf{s}$ ) after CNO treatment (Exp \#1). 
t, Basal CORT levels in the indicated groups on post-stress day 22 ( $n=10$ per group).

$\mathbf{u}-\mathbf{x}$, Behavioral performance in the SIT (u), SPT (v), TST (w), and FST (x) for the indicated groups (Exp \#2).

Data are mean \pm SEM. Gray circles represent individual data points. *, difference compared to control; \#, difference compared to CRST. * $\#, p<0.05 ; * \star, \# \#, p<0.01$ (Student's t-test; One-way ANOVA followed by Newman-Keuls post-hoc test). See Supplementary Table 4 for statistical details.

Fig. 10

Fig. 10 A summary and hypothetical model for the recovery of adaptively changed stress gains by treatment with strategic behavioral stress or GC.

a, Chronic stress produces adaptive changes in the brain, and cumulative effects of chronic stress cause persistent depressive behavior. Repeated treatment with a short sequence of behavioral stress or GC in mice subjected to chronic stress reverses the stress-induced adaptive changes and rescues depressive behavior.

b, The schematic presentation of the brain with normal activity of PL outputs (PLàNAcc, PLàdBNST, and PLàBLA) and the limbic system, and normal activity of the HPA axis including basal GC release at a normal circadian cycle and its feedforward effects on the PL.

c,d, Chronic stress produces the PL overstimulated primarily due to increased GC (c), which results in genome-wide gene expression alteration (d). The reversal of the stress-induced changes by RS5 is included here. As a result, the neural activities of PL outputs (PLàNAcc, PLàdBNST, and PLàBLA) and the limbic system are downregulated or altered, and the basal GC release is enhanced and trailed off from a normal circadian cycle (c). The expression levels of GR, GABAR, NMDAR, p-CaMKIla and Fkbp5 in the PL are changed by chronic stress, and their physiological effects on depressive behaviors are characterized in the present study (d).

e, Repeated treatment with behavioral stress or GC reverses the stress-induced altered neural activities of PL outputs, the altered gene expression profiles, the altered activity of the HPA axis including basal GC release, and the altered feedforward GC effects on the PL.

\section{Extended Data Fig. 1}

\section{Extended Data Fig. 1 Dosing analysis for the repeatability of weak stress that produces anti-depressive effects.}

a, Experimental design. CRST mice were treated with daily 5-min restraint for 3, 5, 7, or 14 days (S5x3d, S5x5d, S5x7d, and S5x14d, respectively) followed by behavioral tests. 
b-f, Time spent in the target chamber in the SIT (b), and immobility time in the TST (c) and FST (d) for the indicated groups. K-Means clustering $(k=2)$ of individuals in the SIT x TST x FST matrix $(\mathbf{e})$ and \% composition of each group in the clusters (f) ( $n=8-10$ per group).

Data are mean \pm SEM. *, difference compared to control; \#, difference compared to CRST. *, \#, $p<0.05$; **, $\# \#, p<0.01$ (One-way ANOVA followed by Newman-Keuls post-hoc test). See Supplementary Table 4 for statistical details.

\section{Extended Data Fig. 2}

\section{Extended Data Fig. 2 Repeated treatment with a short sequence of behavioral stress produces anti- depressive effects in ICR mice.}

a,b, Experimental design (a). Pregnant ICR females were exposed to daily 2-h restraint from E8.5 until delivery (maternal stress or MS group), and their pups (MS pups) were grown to adulthood. Pregnant normal ICR females with no stress (normal or $\mathrm{N}$ group) and their pups ( $\mathrm{N}$ pups) were prepared as controls. Beginning at 7 weeks of age, the MS pups and $\mathrm{N}$ pups were randomly allocated to receive CRST or CRST+RS5 for 14 days (b).

$\mathbf{c}-\mathbf{h}$, Time spent in the target chamber in the SIT (c), sucrose preference in the SPT (d), and immobility time in the TST (e) and FST (f) for the indicated groups, and their controls. PCA and K-Means cluster analysis of individual animals in the SIT x SPT x [TST x FST] matrix (g) and \% composition in each cluster (h). CRST-treated N and MS groups were shifted from the cluster containing CRST mice to the cluster containing the control. The TST and FST components were transformed in a linear dimension by PCA (PVE; 75.6\%) ( $n=9-10$ per group).

Data are mean \pm SEM. *, difference compared to control; \#, difference compared to CRST. *, \#, $p<0.05$; **, $\# \#, p<0.01$ (One-way ANOVA followed by Newman-Keuls post-hoc test). See Supplementary Table 4 for statistical details.

\section{Extended Data Fig. 3}

\section{Extended Data Fig. 3 Pharmacological suppression of HPA axis activation blocks the antidepressive effects of RS5.}

a, Experimental design. Mice were treated with CRST, followed by daily 5-min restraint 30 min after NBI27914 or RU486 injection for indicated days, and subsequent behavioral tests.

b-h, Basal serum CORT levels in the indicated groups (b) ( $n=7-8$ per group). Behavioral performance in the SIT (c), SPT (d), TST (e), and FST (f) for the indicated groups. K-Means clustering of individuals in the SIT x SPT x [TST x FST] matrix $(\mathbf{g})$ and \% composition of each group in the clusters $(\mathbf{h})$. The TST and FST 
components were transformed into a one-dimensional variable using PCA (PVE; 74.4\%) ( $n=7-10$ per group).

Data are mean \pm SEM. *, difference compared to control; \#, difference compared to CRST; + , difference compared to CRST+RS5; @, difference compared to the indicated group. *, $\#,+, @, p<0.05 ; \star \star, ~ \# \#,++$, $@ @, p<0.01$; n.s., not significant (One-way ANOVA followed by Newman-Keuls post-hoc test). See Supplementary Table 4 for statistical details.

\section{Extended Data Fig. 4}

\section{Extended Data Fig. 4 Low-dose CORT treatment activates the brain regions regulating stress coping in CRST mice.}

a, Experimental design. Mice were subjected to CRST, followed by CORT injection (blue arrows) at 0.1, 0.5, or $1.0 \mathrm{mg} / \mathrm{kg} /$ day for 7 days and then sacrificed $25 \mathrm{~min}$ after an additional CORT injection on post-stress day 8. Red arrow, time point for tissue prep.

b, c-Fos expression levels induced by CORT injection in the PL, BLA, NAcc, vSub, dBNST, vBNST, and PVN ( $n=4-6$ per group).

c, Photomicrographs showing c-Fos expression in the PL, BLA, NAcc, vSub, dBNST, vBNST, and PVN (red boxes) of the CRST control, CRST+CORT $(0.1 \mathrm{mg} / \mathrm{kg}), \mathrm{CRST}+\mathrm{CORT}(0.5 \mathrm{mg} / \mathrm{kg})$, and CRST+CORT (1.0 $\mathrm{mg} / \mathrm{kg}$ ) groups.

The details are shown in Supplementary Table 1. Data are mean \pm SEM. *, $* *$, difference compared to control. ${ }^{*}, p<0.05 ;{ }^{* *}, p<0.01$ (one-way ANOVA followed by Newman-Keuls post-hoc test). See Supplementary Table 4 for statistical details.

\section{Extended Data Fig. 5}

\section{Extended Data Fig. 5 Analysis of gene expression profiles and protein-protein interaction networks in the PL of mice exposed to CRST and RS5 treatment.}

a, Serial $K$-Means clustering was used to group the 986 identified genes into featured clusters. Each increase in $k$ value added a new cluster, and its members were mostly supplied from the largest cluster. The cognate inter-clusters are marked with the same color code. C, cluster. Concerning the classification with $k=8$, cluster 1 (gray) contained 622 genes; cluster 2 (scarlet) contained 71 genes; cluster 3 (yellow) contained 39 genes; cluster 4 (light green) contained 29 genes; cluster 5 (orange) contained 28 genes; cluster 6 (blue) contained 27 genes; cluster 7 (green) contained 24 genes; and cluster 8 (violet) contained 19 genes. 
b, Functional protein-protein interaction (PPI) networks constructed with the 237 genes that belonged to clusters 2 to 8 . The members in each cluster are coded with the same colors as indicated above (a).

c, In the classification with $k=8$, clusters $1,2,3,4,6$, and 8 are shown with the number of cluster members and selective modules representing specific GO terms. Clusters 5 and 7 are shown in Fig. 4 d,e.

\section{Extended Data Fig. 6}

\section{Extended Data Fig. 6 RS5 and low-dose CORT treatment upregulates the reduced expression of NMDAR subunits in the PL of CRST mice.}

a, Transcript levels of the NMDA receptor subunits NR1, NR2A, and NR2B in the PL of CON, CRST, CRST + RS5, and CRST + CORT ( $0.1 \mathrm{mg} / \mathrm{kg}$ ) groups. Sample groups were prepared from the experiments depicted in Fig. $4 \mathrm{~g}, \mathrm{~h}(n=7-12$ per group)

b-d, Immunofluorescence staining of NR1 (green) expression in the PL for the indicated groups (b), and quantification levels of NR1/DAPI (c) relative to DAPI intensity (d) $(n=4-6$ animals per group; $\mathrm{n}=209$ 359 per group). DAPI, blue.

$\mathbf{e}-\mathbf{h}$, Immunofluorescence staining of NR2A (red) and NR2B (green) expression in the PL for the indicated groups (e). DAPI, blue. Quantification levels of NR2A/DAPI (f) and NR2B/DAPI (g), and DAPI intensity (h) in individual cells ( $n=4-6$ animals per group; $n=231-402$ per group).

Data are mean \pm SEM. * , difference compared to control; \#, difference compared to CRST. *, \#, $p<0.05$, **, $\# \#, p<0.01$ (One-way ANOVA followed by Newman-Keuls post-hoc test). See Supplementary Table 4 for statistical details.

\section{Extended Data Fig. 7}

\section{Extended Data Fig. 7 Chemogenetic activation of PL glutamatergic neurons in CRST mice improves stress-induced depressive behaviors.}

a-f, Experimental design (a). Mice injected in the PL with the AAV8-CaMKIlla-hM3D(Gq)-mCherry excitatory vector were subjected to CRST, followed by CNO (red arrow) or Veh (black arrow) injection. Injection of the AAV8 vector and resulting mCherry expression in the PL (b). CNO-stimulated c-Fos induction in the PL of mice carrying AAV8-CaMKIla-hM3D(Gq)-mCherry (c and d) and AAV8-hSyn$\mathrm{hM} 3 \mathrm{D}(\mathrm{Gq})$-mCherry (e and f) (Exp \#1). CNO doses, $0.1,1.0$, or $3.0 \mathrm{mg} / \mathrm{kg}$.

$\mathbf{g}-\mathbf{k}$, Behavioral performance in the SIT (g), TST (h), and FST (i) for the indicated groups on post-stress day 1 (Exp \#2-1). K-Means clustering of individuals in the SIT x TST x FST matrix (j) and \% composition of each group in the clusters ( $\mathbf{k})(n=8$ per group). 
I-p, Behavioral performance in the SIT (I), TST (m), and FST (n) for the indicated groups after CNO washout on post-stress day 15 (Exp \#2-2). K-Means clustering of individuals in the SIT x TST x FST matrix (o) and \% composition of each group in the clusters ( $\mathbf{p})$ ( $n=8$ per group).

q. Basal serum CORT levels in the indicated groups on post-stress day 22 ( $n=8$ per group).

Data are mean \pm SEM. *, difference compared to control; \#, difference compared to CRST. *, \#, $p<0.05$, **, $\# \#, p<0.01$ (One-way ANOVA followed by Newman-Keuls post-hoc test). See Supplementary Table 4 for statistical details.

Extended Data Fig. 8

Extended Data Fig. 8 PL neurons projecting to the dBNST, NAcc and BLA are visualized by labeling with AAV-CaMKIla-GFP.

$\mathbf{a}-\mathbf{f}$, Experimental design (a). The anterograde tracer AAV-CaMKIla-GFP was injected into the PL region (b and $\mathbf{c}$ ), and two weeks later, subject mice were sacrificed and GFP expression in the brain was examined. Photomicrographs showing GFP signals in the dBNST (d), NAcc (e), BLA (f), and PVN (g) regions. High magnification of the area marked with a box is indicated on the right panel of each region. dBNST, dorsal BNST, CaA, central amygdala; BLA, basolateral amygdala; NAcc, NAc core; PVN, paraventricular nucleus of the hypothalamus.

h-I, Experimental design (h). The retrograde tracer, cholera toxin subunit B-488 (CTB488), was injected into the dBNST (i and j). A week later, mice were sacrificed and CTB488 expression in the brain was examined. Photomicrographs showing CTB488 expression in the PL (k) and ventral subiculum (vSub) (I). High magnification of the area marked with a box is indicated on the right side.

\section{Methods}

\section{Animals}

Seven-week-old male C57/BL6 mice were purchased from Daehan BioLink (Eumsung, Chungbuk, Republic of Korea). Eight-week-old male and female ICR (CD1) mice were purchased from OrientBio. Inc. (Seongnam, Gyeonggi, Republic of Korea) and used as breeders. All mice were housed in pairs in standard clear plastic cages in a temperature $\left(23-24^{\circ} \mathrm{C}\right)$ - and humidity $(50-60 \%)$-controlled room, with food and drinking water available ad libitum. The animal room was maintained on a 12-hour light/dark cycle (light on at 7 AM) in a specific-pathogen-free environment. Animal experiments were performed in accordance with the animal care guidelines of Ewha Womans University (IACUC 16-018).

\section{Short-term restraint stress}


Short-term restraint was delivered to give weak restraint stress. Mice were individually placed in a 50-ml polypropylene conical tube with many ventilation holes and restrained for $5 \mathrm{~min}, 10 \mathrm{~min}, 15 \mathrm{~min}$, or the indicated time beginning at $10 \mathrm{AM}$, and this treatment was repeated for the indicated number of days. After each restraint session, the mice were returned to their home cages.

\section{Chronic restraint stress (CRST)}

CRST was carried out as described previously ${ }^{58}$. In brief, mice were individually restrained in a wellventilated $50-\mathrm{ml}$ conical tube for $2 \mathrm{~h}$ daily from $10 \mathrm{AM}$ to $12 \mathrm{PM}$, and this procedure was repeated for 14 days. After each daily restraint session, the mice were placed in their home cages with free access to food and water.

\section{Chronic social defeat stress (CSDS)}

CSDS was carried out as described previously ${ }^{59,60}$. Briefly, mice were individually exposed to a novel ICR aggressor for 10 minutes to produce physical defeat stress and then housed for the remainder of the day in a compartment of the ICR aggressor's cage partitioned with a perforated acrylic divider. This procedure was repeated for 10 days with a different ICR aggressor each day. ICR aggressors were preselected: if ICR aggressors showed $\leq 30 \mathrm{~s}$ attack latencies on three consecutive screening tests, or if any aggressor exhibited overly aggressive attacks during social defeat exposure, they were excluded from the next experiment. Stress-susceptible and resilient mice were selected on the basis of sociability in the open-field version of the SIT on day 11 by following the procedure described previously 59,60 .

\section{Maternal stress (MS) and postnatal stress treatments}

Male and female ICR mice at 8-9 weeks of age were crossed, and pregnant females were randomly assigned to the maternal stress (MS) or normal (N) control group. MS pregnant females were treated with restraint daily for $2 \mathrm{~h}(10 \mathrm{AM}-12 \mathrm{PM}$ ) from 8.5 days post coitus (dpc) to delivery (at $19.5-20.5 \mathrm{dpc}$ ). Normal $(\mathrm{N})$ pregnant females were maintained in parallel to the MS females. The offspring of both groups were weaned at postnatal day 20 (PN20) and reared in pairs with the same sex in cages under standard conditions. For mouse groups assigned for CRST treatment, on PN49, offspring from normal (N) control mothers or MS mothers were housed in same-sex pairs from different litters to prevent possible litter effects. They were then treated with daily 2-h restraint for 14 days (PN50 - PN63). Afterward, the half of the CRST-treated mice were subjected to the RS5 regimen, and the remaining half was used as the CRST-treated group. 


\section{Immunohistochemistry}

Immunohistochemistry was performed as described previously ${ }^{38,39}$. Briefly, mice were perfused with $4 \%$ paraformaldehyde via the trans-cardiac method and isolated brains were postfixed at $4^{\circ} \mathrm{C}$ overnight. Each brain was coronally sectioned into 40- $\mu$ m thicknesses using a vibratome (VT1000S, Leica Instruments, Nussloch, Germany). The collected sections were blocked for $1 \mathrm{~h}$ with $4 \%$ bovine serum albumin in phosphate buffered saline (PBS) containing $0.1 \%$ Triton X-100 (PBST) and then incubated with primary antibody at $4^{\circ} \mathrm{C}$ overnight. After washing, sections were reacted for 90 min with biotinylated secondary antibody: anti-rabbit IgG (BA-1000, Vector Laboratories, Burlingame, CA, USA) or anti-mouse IgG (BA9200, Vector Laboratories) diluted at 1:200 in PBST. Signals were visualized using an ABC Elite kit (PK6200 , Vector Laboratories).

For the analysis of $c$-Fos expression induced by weak restraint stress, CRST mice were treated with a single 5-min restraint or 15-min restraint (S5 and S15, respectively) on post-stress day 8 , and they were sacrificed, $20 \mathrm{~min}$ and $10 \mathrm{~min}$ later, respectively. Other groups of CRST mice were treated with 5-min restraint or 15-min restraint for 8 days (S5x8d and S15x8d, respectively), and sacrificed 20 min and 10 min, respectively, after the last restraint.

For the analysis of $c$-Fos expression induced by low-dose CORT injection, CRST mice were given CORT injections at $0.1,0.5$, or $1.0 \mathrm{mg} / \mathrm{kg} /$ day for 7 days and sacrificed $25 \mathrm{~min}$ after an additional CORT injection on post-stress day 8.

The level of c-Fos expression in specific brain regions was quantified using an Olympus BX 51 microscope equipped with a DP71 camera and MetaMorph Microscopy Automation \& Image Analysis software (Molecular Devices, Sunnyvale, CA, USA) and also using a six-point rating scale, as described previously ${ }^{61}$. The $c$-Fos expression level was assessed by a numerical grade of $0-5$ scales; 0 assigned for $0-50$ c-Fos-positive cells $/ \mathrm{mm}^{2}$ in a counting region; +1 for $51-100$ c-Fos-positive cells $/ \mathrm{mm}^{2} ;+2$ for $101-200$ c-Fos-positive cells/mm²; +3 for $201-350$ c-Fos-positive cells $/ \mathrm{mm}^{2} ;+4$ for $351-600 \mathrm{c}$-Fospositive cells $/ \mathrm{mm}^{2}$; and +5 for $>600$ c-Fos-positive cells $/ \mathrm{mm}^{2}$. The average $c$-Fos expression score in each region was rounded to the nearest tenth.

The primary antibodies used were anti-c-Fos (sc-52 and sc-271243, Santa Cruz Biotechnology, CA, USA), anti-Glu4 (G9282, Sigma-Aldrich, MO, USA), anti-GAD67 (MAB5406, EMD Millipore, CA, USA), anti-GR (sc136209, Santa Cruz Biotechnology; 12041S, Cell Signaling Technology, MA, USA), anti-Fkpb5 (GTX84491, Genetex, CA, USA), anti-NR1 (sc-1467, Santa Cruz Biotechnology), anti-NR2A (sc-9056, Santa Cruz Biotechnology), anti-NR2B (ab93610, Abcam, Cambridge, UK), anti-pCaMKIla (Thr286) (sc-12886, Santa Cruz Biotechnology), and anti-pERK1/2 (Thr202/Tyr204) (4370s, Cell Signaling Technology). For immunofluorescence staining, the following fluorescence-tagged secondary antibodies were used: antirabbit IgG DyLight488 (DI-1488; 1:500, Vector Laboratories), anti-rabbit IgG DyLight594 (DI-1094; 1:500, Vector Laboratories), anti-mouse IgG DyLight488 (DI-2488; 1:500, Vector Laboratories), anti-mouse IgG DyLight594 (DI-2594; 1:500, Vector Laboratories), and anti-goat IgG-FITC (sc-2356, 1:200, Santa Cruz 
Biotechnology). Stained sections were mounted with antifade mounting solution containing DAPI (H1200; Vector Laboratories). Stained immunofluorescence images were analyzed using an Olympus BX 51 microscope equipped with a DP71 camera and the MetaMorph program (Molecular Devices).

\section{Real-time PCR analysis}

Real-time PCR was carried out as described previously ${ }^{38,39}$. Total RNA was purified from brain tissue using TRIzol reagent (15596018, Invitrogen Life Technologies, Carlsbad, CA, USA) and was treated with DNase I (M610A, Promega, MO, USA) to eliminate genomic DNA contamination. Reverse transcription of total RNA $(2 \mu \mathrm{g})$ was conducted in a volume of $20 \mu \mathrm{l}$ using a reverse transcription system (A3500, Promega, MO, USA). Real-time PCR was performed with $10 \mu \mathrm{l}$ of $2 X$ iQ ${ }^{\text {TM }}$ SYBR Green Supermix (\#170882, Bio-Rad Laboratories, Foster City, CA, USA), $1 \mu \mathrm{l}$ each of $5 \mathrm{pmol} / \mu \mathrm{l}$ forward and reverse primers, and $4 \mu \mathrm{l}$ of complementary DNA (CDNA) (1/8 dilution of the conversion) in a total volume of $20 \mu$ using a CFX 96 Real-Time PCR System Detector (Bio-Rad Laboratories).

The primer sets used were 5'-CACCTACCAAGGGAGGAGAA-3' and 5'-GCAGGCAGGACGACAGAG-3' for CRH, 5'-GCTCAACACTACGCTCTC-3' and 5'-CTTGGGCAGTTCTGGAAG-3' for AVP, 5'-

GGAATAGGTGCCAAGGGTCT -3' and 5'-CACGTCAGCACCCCATAATG -3' for Nr3c1(GR), 5'CACTTGAGTTCCTTTCCGCC-3' and 5'-TCCACACACCAAGCAGATCT-3' for Nr3c2(MR), 5'AGCCGTACTTCACTCTCCGT-3' and 5'-AAGCCCCATCCTTTATGGCG-3' for Fkbp5, 5'GGCTCCGAAGTTGATAGCAG-3' and 5'-GCTTGCCTCAATCTCTGGTC-3' for Fkbp4, 5'GCCTGTTTGGAAAGGTGGTA-3' and 5'-TCCACCTTGAAAGGCAAATC-3' for Hsp90aa1, 5'ATGACTGGGAGGACCACTTG-3' and 5'-CACCTCCAGCTTCTGCCTAC-3' for Hsp90ab1, 5'AGTGGAGATGAAAGGCACGA-3' and 5'-GACTTGGGTAGAGCTCCACA-3' for Dusp1, 5'CCGGAATCTTCTGAGAGCAC-3' and 5'-CCTCTGGTTCAAAGGCTGTC-3' for CaMKIla, 5'CATCAGCACTCTCATTCGCC-3' and 5'-AGGAAAATGGGCTCTGACGA-3' for Mapk3(ERK1), 5'TGATCAGCAGACAACCGAAG-3' and 5'-GTATTGATGCTTGGCCCACT-3' for Mapk1(ERK2), 5'TTCACAGAAGTGCGATCTGG-3' and 5'-GGACAGGGACACATTTTGCT-3' for NR1, 5'CTCCTTGGTCACCACCATCT-3' and 5'-TCAGCTGGACCTGTGTCTTG-3' for NR2A, 5'GTGAGAGCTCCTTTGCCAAC-3' and 5'-AGGGTAGAGCGACTTGCTGA-3' for NR2B, 5'AAGGACCCATGACAGTGCTC-3' and 5'-GTGCCATCCTCTGTGATACG-3' for GABRa1, 5'AATGGAGGAGAGGGAGCAGT-3' and 5'-CCACCTTCTTGGACACCATC-3' for GABRß2, and 5'GCTGCCATCTGTTTTACGG-3' and 5'-TGACTGGTGCCTGATGAACT-3' for L32.

\section{Corticosterone measurement}

Serum corticosterone levels were measured as described previously ${ }^{39}$. Mice were anesthetized by intraperitoneal injection (300 $\mathrm{LL} /$ mouse) with $2.5 \%$ avertin (2,2,2-tribromoethanol; $300 \mathrm{mg} / \mathrm{kg})$ (T48402, 
Sigma-Aldrich) in tert-amyl alcohol (24,048-6, Sigma-Aldrich) diluted to $2.5 \%$ avertin with saline. Blood was collected from the abdominal aortas of sacrificed mice in the morning (8 AM - 12 PM), and serum was obtained by centrifugation at $3,000 \mathrm{~g}$ and $4^{\circ} \mathrm{C}$ for $15 \mathrm{~min}$ and then stored at $-80^{\circ} \mathrm{C}$ until use. Each diluted serum sample $(10 \mu \mathrm{l})$ was mixed on a 96 -well plate with an equal volume of a steroid displacement reagent solution and sera diluted at 1: 40 or 1:80 in the ELISA assay buffer provided in the corticosterone ELISA kit (ADI-901-097, Enzo Life Sciences, NY, USA). The reaction was incubated for $2 \mathrm{~h}$ at room temperature on an orbital shaker rotating at $120 \mathrm{rpm}$. The reaction mixture in each well was discarded, and the plate was rinsed with washing buffer. The pNpp substrate solution provided in the kit was added at $200 \mu \mathrm{l} /$ well, and the plate was incubated for $1 \mathrm{~h}$ at room temperature without shaking. Finally, the absorbance at $405 \mathrm{~nm}$ was measured using a spectrofluorometer (SpectraMax ${ }^{\circledR} \mathrm{M} 5$, Molecular Devices, Sunnyvale, CA, USA).

\section{Chemogenetic manipulation of the activity of specific neurons}

Chemogenetic manipulation of neurons was carried out using a DREADDs system as described previously ${ }^{62}$. Mice were anesthetized with a mixture of ketamine and xylazine (3.5:1). To activate PL neurons, the CaMKIla promoter-driven hM3D viral vector AAV8-CaMKIIa-hM3D(Gq)-mCherry $\left(3 \times 10^{10}\right.$ viral particles/ml) or the hSyn promoter-driven hM3D viral vector AAV8-hSyn-hM3D $(\mathrm{Gq})$-mCherry $\left(4.3 \times 10^{10}\right.$ viral particles $/ \mathrm{ml}$ ) was bilaterally injected into the PL. To inhibit PL neurons, the CaMKIla promoter-driven hM4D viral vector AAV8-CaMKIla-hM4D(Gi)-mCherry $\left(1.6 \times 10^{10}\right.$ viral particles $\left./ \mathrm{ml}\right)$ was bilaterally injected into the PL. After 7 recovery days, mice were treated with CRST $(2 \mathrm{~h} \times 14 \mathrm{~d})$. They were then injected with vehicle or clozapine-N-oxide (CNO) $(0.1,1$, or $3 \mathrm{mg} / \mathrm{kg}) 30 \mathrm{~min}$ before treatment with 5 -min restraint according to the experimental design.

To label the PLàdBNST circuit, the viral vector AAV8-hSyn-DIO-hM4D(Gi)-mCherry $\left(3 \times 10^{10}\right.$ viral particles $/ \mathrm{ml}$ ) was bilaterally injected into the PL (AP, +2.0; $\mathrm{ML}, \pm 0.23 ; \mathrm{DV},-2.0 \mathrm{~mm}$ ). Immediately afterwards, the retrograde transport-promoting Cre vector AAV8-EF1a-mCherry-IRES-WGA-Cre $\left(1.5 \times 10^{10}\right.$ viral particles $/ \mathrm{ml}$ ) was injected into the dBNST (AP, $+0.25 ; \mathrm{ML}, \pm 1.15 ; \mathrm{DV},-4.2 \mathrm{~mm}$ ) as depicted in the experimental design. For chemogenetic inhibition of PLàdBNST neurons, they were injected with vehicle or CNO (3 mg/kg, i.p.) $30 \mathrm{~min}$ before treatment with 5 -min restraint, as depicted in the experimental design. The injection volume and injection rate for each target site were as follows: for $\mathrm{PL}, 0.5 \mu \mathrm{l}$ at a rate of $0.25 \mu \mathrm{l} / \mathrm{min}$; and for $\mathrm{dBNST}, 0.3 \mu \mathrm{l}$ at a rate of $0.15 \mu \mathrm{l} / \mathrm{min}$. The injection needle was left in the site for 5 min to ensure adequate delivery and was withdrawn slowly over the course of 5 min.

To label the PLàNAcc or PLàBLA circuit, the viral vector AAV8-hSyn-DIO-hM4D(Gi)-mCherry $\left(3 \times 10^{10}\right.$ viral particles $/ \mathrm{ml}$ ) was bilaterally injected into the PL (AP, +2.0; $M L, \pm 0.23 ; D V,-2.0 \mathrm{~mm}$ ). Immediately afterwards, the retrograde transport-promoting Cre vector AAV8-EF1 a-mCherry-IRES-WGA-Cre $\left(1.5 \times 10^{10}\right.$ viral particles $/ \mathrm{ml}$ ) was injected into the NAc (AP, $+1.65 ; \mathrm{ML}, \pm 1.15 ; \mathrm{DV},-5.15 \mathrm{~mm})$ or BLA (AP, -1.3; $\mathrm{ML}$, \pm 3.35 ; DV, $-5.2 \mathrm{~mm}$ ) as depicted in the experimental design. 
AAV8-CaMKIla-hM3D(Gq)-mCherry, AAV8-CaMKIla-hM4D(Gi)-mCherry, AAV8-hSyn-hM3D(Gq)-mCherry, and AAV8-hSyn-DIO-hM4D(Gi)-mCherry were obtained from Dr. Bryan Roth (Addgene: plasmids \#50476, \#50477, \#50474, and \#44362, respectively). AAV8-EF1a-mCherry-IRES-WGA-Cre was purchased from UNC Vector Core (University of North Carolina, NC, USA). Viral vectors were aliquoted upon arrival and stored at $-80^{\circ} \mathrm{C}$ until use. Each viral vector was diluted in 1 x PBS $\left(13.7 \mathrm{mM} \mathrm{NaCl}, 0.27 \mathrm{mM} \mathrm{KCl}, 0.8 \mathrm{mM} \mathrm{Na}{ }_{2} \mathrm{HPO}_{4}\right.$, $\left.0.2 \mathrm{mM} \mathrm{KH}_{2} \mathrm{PO}_{4}, \mathrm{pH} 7.4\right)$.

\section{Neural circuit mapping with anterograde and retrograde tracers}

Mice were anesthetized with ketamine and xylazine (3.5:1). To label the anterograde projections of PL neurons, AAV2/1-CaMKIla-EYFP (1.0 × $10^{9}$ viral particles/ml) (AV-1-PV1917, University of Pennsylvania, $\mathrm{PA}$, USA) was stereotaxically injected into the PL (AP, +2.0; ML, -0.23 ; DV, $-2.0 \mathrm{~mm}$ ) with a volume of $0.5 \mu \mathrm{l}$ at the rate of $0.25 \mu \mathrm{l} / \mathrm{min}$ using a $30-\mathrm{G}$ needle. Two weeks after the stereotaxic injection, the brains were prepared after perfusion with $4 \%$ paraformaldehyde via a trans-cardiac method. To label the neuronal afferents to the dBNST, Alexa Fluor 488-conjugated cholera toxin subunit B (CTB) $(0.1 \% \mathrm{w} / \mathrm{v})(\mathrm{C} 34755$, Molecular Probes, Eugene, OR, USA), a retrograde tracer, was stereotaxically injected into the dBNST (AP, $+0.25 ; \mathrm{ML},-1.15 ; \mathrm{DV},-4.2 \mathrm{~mm}$ ) in a volume of $0.3 \mu \mathrm{l}$ at a rate of $0.15 \mu \mathrm{l} / \mathrm{min}$ using a $30-\mathrm{G}$ needle. One week after the stereotaxic injection, the brains were prepared after perfusion with $4 \%$ paraformaldehyde as described above. The brains were coronally sectioned into $40-\mu \mathrm{m}$ thicknesses with a vibratome (Leica VT 1000S; Leica Instruments, Nussloch, Germany). Fluorescence images were collected and analyzed using an Olympus BX 51 microscope equipped with an X-cite 120 fluorescence illuminator (EXFO Life Science \& Industrial Division, Ontario, Canada), a DP71 camera (Olympus), and MetaMorph Microscopy Automation \& Image Analysis software (Molecular Devices, Sunnyvale, CA, USA).

\section{Microinjection of small-interfering RNA (siRNA)}

Stereotaxic injection of siRNA was carried out as described previously ${ }^{38,63}$. Briefly, mice were anesthetized with a mixture of ketamine hydrochloride $(50 \mathrm{mg} / \mathrm{ml})$ and xylazine hydrochloride $(23.3 \mathrm{mg}$ $/ \mathrm{ml})(3.5: 1)$ at a dose of $2.5 \mathrm{ml} / \mathrm{g}$ body weight. One volume of diluted $(50 \mathrm{ng} / \mathrm{ml})$ siRNA was mixed with 2.5 volumes of Neurofect transfection reagent (T800075, Genlatis, San Diego, CA, USA) and 0.5 volumes of $50 \%$ sucrose and incubated for $20 \mathrm{~min}$ before injection into the brain. A total of $0.5 \mu \mathrm{l}$ of a mixture containing $5.94 \mathrm{ng}$ of siRNA was injected into each side of the PL (AP, $+2.0 ; \mathrm{ML}, \pm 0.23 ; \mathrm{DV},-2.0 \mathrm{~mm}$ ) at a rate of $0.25 \mu \mathrm{l} / \mathrm{min}$ using a $30-\mathrm{G}$ needle. Behavioral tests were performed between 48 and $72 \mathrm{~h}$ after the injection of siRNA.

The siRNAs used were siRNA-control (SN-1012), siRNA-CaMKIlla (\#12322, NM_177407.2, NM_009792.1), siRNA-MAPK3 (ERK1) (\#26417, NM_011952.2), siRNA-MAPK1 (ERK2) (\#26413, NM_011949.3, 
NM_001038663.1), or siNr3c1(GR) (\#14815, NM_008173.3), all of which were purchased from Bioneer Co. (Daejeon, Korea).

\section{Drug infusion through a cannula}

Cannula implantation and drug infusion were performed as described previously ${ }^{63}$. In brief, mice were anesthetized with ketamine and xylazine (3.5: 1). A 26-gauge guide cannula (C315G/SPC, Plastics One, Bilaney, UK) was stereotaxically implanted into the PL (AP, +2.0; $\mathrm{ML},+0.23 ; \mathrm{DV},-2.0 \mathrm{~mm})$. The cannula was fixed in place by applying Super-Bond $C \& B^{\text {TM }}$ dental adhesive (Sun Medical, Moriyama, Japan) to the surface of the mouse skull around the cannula base. After $15 \mathrm{~min}$, dental cement (Vertex-Dental, AA Zeist, The Netherlands) was applied to fix the cannula to the skull surface, and then sealed with a dummy cannula (C313DC, Plastics One). The mice had more than 7 recovery days. While the mice were anesthetized with $1.4 \%$ isoflurane in a gas mixture of $70 \%$ nitrous oxide and $30 \%$ oxygen, drugs were infused into the PL in a volume of $0.5 \mu$ via a 33-gauge internal cannula (C315I, Plastics One).

KN62 (2.5 nmol/injection; 12142, Sigma-Aldrich) was delivered into the PL in a volume of $0.5 \mu \mathrm{l}$ at a rate of $0.25 \mu \mathrm{l} / \mathrm{min}$ through cannulation.

\section{Drug administration}

Imipramine (10899, Sigma-Aldrich), corticosterone (C2505, Sigma-Aldrich), NBI27914 hydrochloride (1591, Tocris Bioscience), RU486 (M8046, Sigma-Aldrich), and Clozapine N-oxide (CNO) dihydrochloride (6329/10, Tocris Bioscience) were diluted in $0.9 \%$ saline and injected intraperitoneally in a volume of 120 $\mu \mathrm{l}$.

\section{Western blot analyses}

Western blot analyses were performed as described previously ${ }^{38,58}$. Briefly, PL tissue ( $n=2-4$ mice) was homogenized in RIPA buffer ( $50 \mathrm{mM}$ Tris- $\mathrm{HCl}, \mathrm{pH} 7.4,150 \mathrm{mM} \mathrm{NaCl}, 0.5 \%$ Nonidet P-40, $0.25 \%$ sodium deoxycholate, $1 \mathrm{mM}$ EDTA, and $0.5 \%$ Triton X-100) containing a protease inhibitor cocktail (11836153001, Roche, Mannheim, Germany). Tissue homogenates loaded at $20 \mathrm{mg}$ of protein/lane were resolved on SDS-PAGE and transferred onto PVDF (1620177, Bio-Rad Laboratories). The membranes were incubated with blocking solution ( $5 \%$ skim milk; 232100, Difco, MI, USA) in TBST (150 mM NaCl, $50 \mathrm{mM}$ Tris- $\mathrm{HCl}$, pH $7.4,0.1 \%$ Tween 20 ) followed by primary antibody diluted in $1 \%$ skim milk at $4^{\circ} \mathrm{C}$ overnight. After washing with 1 x TBST 6 times for 10 min each time, the membranes were incubated with secondary antibody for $1 \mathrm{~h}$. The secondary antibodies used were anti-rabbit IgG-HRP (sc-2004, Santa Cruz Biotechnology) and anti-mouse IgG-HRP (sc-2005, Santa Cruz Biotechnology) diluted 1:2,000 in 1\% skim milk. After washing, 
specific signals on the blots were visualized using an enhanced chemiluminescence kit (ELPIS-Biotech, Daejeon, Korea). Western blot images were quantified using Image $\mathrm{J}$ analysis software (NIH Image, Bethesda, MD, USA).

The primary antibodies were anti-p-CaMKIla (Thr286) (sc-12886, Santa Cruz Biotechnology), CaMKIla (A1) (sc-13141, Santa Cruz Biotechnology), anti-p-ERK1/2 (Thr202/Tyr204) (4370s, Cell Signaling Technology), anti-ERK1/2 (MK1) (sc-135900, Santa Cruz Biotechnology), and anti- $\beta$-actin (C4) (sc-47778, Santa Cruz Biotechnology).

\section{Adrenalectomy}

Adrenalectomy (ADX) was carried out as described previously ${ }^{64}$. Mice were anesthetized using a mixture of ketamine and xylene (3.5: 1). Surgical tools were pre-sterilized with Bromosept (DAESUNG Microbiological Labs. Co., Kyonggi, Republic of Korea). Mice were placed prone on the surgical bed. The dorsal side of the body was covered with a $10 \%$ of povidone iodine solution (\#261, Sungkwang, Chungnam, Republic of Korea). The left side of the back was grabbed with a forceps (\#5, Fine Science Tools Inc., North Vancouver, B.C. Canada), and the skin and peritoneum were sequentially incised with micro-dissecting scissors (S3271, Sigma-Aldrich). Then, the adrenal gland was gripped with a forceps (\#5, Fine Science Tools Inc.) and cut off with a scalpel blade (S2646, Sigma-Aldrich) assembled on a scalpel handle (S2896, Sigma-Aldrich). The peritoneum and skin were sequentially stitched with silk suture 4/0-18 mm (SK434, Ailee Company Limited, Busan, Republic of Korea). The right side operation followed the same procedure as the left. Adrenalectomized mice were subsequently given $0.9 \%$ saline solution instead of drinking water, but they were given no corticosterone replacement. Sham-operated mice underwent the same surgical procedure, but although the adrenal glands were grabbed with the forceps, but they were not removed. After 7 recovery days, half of the ADX and sham-operated mice were subjected to RS5 treatment, and the remaining mice served as controls.

\section{Microarray analysis}

Microarray analysis was carried out as described previously ${ }^{38}$. In brief, two independent sets of control (CON), CRST, and CRST+RS5 groups were prepared. Mice were subjected to CRST treatment followed by RS5, and then sacrificed on post-stress day 22. Total RNA was extracted from the pooled PL of the $6-7$ animals in each group using Trizol (Invitrogen Life Technologies), followed by purification using RNeasy columns (Qiagen, Valencia, USA). The purified RNA had an A260/280 ratio of $1.9-2.1$ as determined by an ND-1000 Spectrophotometer (NanoDrop, Wilmington, DE, USA) and an Agilent 2100 Bioanalyzer (Agilent Technologies, Palo Alto, CA, USA). 
Total RNA (550 ng for each) was converted to first- and second-strand cDNA by in vitro transcription. The cDNA was then used to synthesize biotin-labeled cDNA samples. Purified biotin-labeled cDNA samples were fragmented by heating them to $94^{\circ} \mathrm{C}$ for $35 \mathrm{~min}$ in array fragmentation buffer. Each of fragmented and biotin-labeled cDNA samples (750 ng) was hybridized to the mouse Ref8 expression v.2 bead array (Illumina, Inc., San Diego, USA) for $16-18 \mathrm{~h}$ at $58^{\circ} \mathrm{C}$, according to the manufacturer's instructions. After washing, the array signals were amplified with Amersham Fluorolink streptavidin-Cy3 (GE Healthcare BioSciences, Little Chalfont, UK) and scanned with an Illumina Bead Array Reader, a confocal scanner according to the manufacturer's instructions.

The quality of hybridization and overall chip array performance, which included the internal quality control probes and the scanned raw data extracted using the Illumina GenomeStudio v2011.1 software (Gene Expression Module v1.9.0) were monitored by Macrogen Inc. (Seoul, South Korea). The microarray signals were converted into log2 scale values and normalized by the quantile method.

Gene expression profiles were analyzed using Multiexperiment Viewer (MeV), version 4.9 (http://mev.tm4.org/). Genes whose expression were up- or down-regulated $\geq 1$.2-fold by CRST (CON vs. CRST) and those changed $\geq 1$. 2 -fold after RS5 treatment of CRST-treated mice (CRST vs. CRST+RS5) were selected. The expression profiles of the 264 CRST-responsive genes were presented from the highest to the lowest value in a heatmap followed by unsupervised alignment with the respective genes from the CRST vs. CRST+RS5 comparison. Independently, the gene expression profiles of the 722 RS5-responsive genes were aligned similarly as above in a heatmap and then overlaid with the respective genes from the CON vs. CRST comparison.

\section{Gene Ontology (GO) enrichment analysis}

GO enrichment analysis was carried out using the STRING (Search Tool for Retrieval of Interacting Genes/Proteins) v11 database ${ }^{31}$ (available at: http://string-db.org/). Of the total $896(264+722)$ genes selected as described in the previous section, 860 genes were assigned to functional groups with biological process (BP) terms, whereas the 36 genes with an unspecified identity were excluded from further analyses. The GO term hierarchy was assigned based on the Mouse Genome Database ${ }^{56}$ (http://www.informatics.jax.org).

Serial $K$-Means clustering was used to group the 860 genes into multiple functional clusters. Each increase in $K$ value added a new cluster which contained members mostly supplied from the largest cluster (cluster 1 ). When $>75 \%$ of the genes in a cluster remained as a cluster in the next serial clustering, those two inter-clusters were considered to be in the same functional group and are therefore marked with the same color code. The retention rate of most cognate inter-clusters was higher than $85 \%$ for grouping into $5-10$ clusters $(k=5-10)$, whereas the retention rate dropped after grouping into 11 or more clusters. 
A functional protein-protein interaction (PPI) network (PPI enrichment p-value, <1.0e-16) consisting of 8 clusters $(k=8)$ were chosen as a representative grouping: Cluster 1 contained the most members (622 genes). However, the members of cluster 1 were poorly interactive and scattered outside the functional networks formed by the remaining 7 clusters, which included 238 genes in total. Therefore, the cluster 1 was excluded from further analyses. Clusters 2 to 8 contained 71, 39, 29, 28, 27, 25 and 19 genes, respectively. Clusters 5 and 7 , which contained 28 and 25 genes, respectively, covered the GO terms "response to stress" and "response to glucocorticoids", along with the cell communication, the estabilishment of localization, and response to endogenous stimuli.

Potential interactors which were missed from the microarray screening but that could exist in the functional PPI networks were sought in the STRING network database. Using an edge confidence of 0.7 and higher to classify nodes in the PPI networks, that effort produced 16 new interactors. Afterwards, functional networks were constructed to include the members of clusters 5 and 7 and the 16 new members. The nodes and edges for interactions with members were color-coded using Cytoscape StringApp v3.7.2 ${ }^{57}$.

Real-time PCR was used to examine the expression profiles of the Nr3c1(GR), Nr3c2(MR), Fkbp5, Fkbp4, Hsp90aa1, and Hsp90ab1 genes, whch were arbitrarily chosen from cluster 7, and the expression of Dusp1, CaMKIla, Mapk3, and Mapk1, which were selected from cluster 5 for the indicated groups.

\section{Behavioral assessments}

Behavioral tests were carried out as described previously ${ }^{38,39}$. In brief, all behavioral tests were recorded with a computerized video tracking system (SMART; Panlab S.I., Barcelona, Spain) or a webcam recording system (HD Webcam \#C210; Logitech, USA). All behavioral tests among groups and within groups were conducted in a randomized fashion or in an alternating manner with respect to test order and position within the testing equipment or test field (e.g., left $v s$. right side, between positions in the test room). CRST-induced persistent behavioral changes, and RS5 effects were verified in a blind manner by two experimenters. All animals were housed in pairs unless otherwise indicated. No bedding change was made the day before the behavioral tests. The behavioral testing room was lit with 20 lux of indirect illumination for the sociability test and 250 lux for the TST and FST. Mice were given 20-30 min to adapt to the behavior testing room prior to starting each behavioral test. The background sound in the testing room was masked with $65 \mathrm{~dB}$ of white noise throughout all behavioral tests. All behavioral tests were performed from 9 AM - 3 PM during the light cycle. After each behavioral test, all parts of the apparatus were cleaned with $70 \%$ ethanol.

\section{Two-chamber social interaction test}


The two-chamber social interaction test (SIT) was developed as a modified version of the three-chamber test ${ }^{58}$ and the U-shaped two-choice field test ${ }^{68}$. In brief, the two-chamber apparatus consisted of two symmetrical chambers ( $26 \times 26 \mathrm{~cm}$ rectangular floor with $40 \mathrm{~cm}$ walls) separated by an intermediate path ( $8 \mathrm{~cm}$ wide $\times 10 \mathrm{~cm}$ long $\times 26 \mathrm{~cm}$ in height), with an empty circular grid cage $(12 \mathrm{~cm}$ in diameter $\times 33 \mathrm{~cm}$ in height) in each chamber. A subject mouse was placed in the middle path and allowed to freely explore the two chambers for $5 \mathrm{~min}$ as habituation. While the subject mouse was returned to its home cage for 2 min, a social target (C57BL/6) was placed in one of the grid cages. As soon as the social target was stabilized, the subject mouse was placed in the middle of the two choice chambers, with a social target on one side and an empty grid cage on the other side, and allowed to freely explore the chambers for 5 $\min$. The time spent and the trajectory taken in each chamber were recorded. The side containing the social target was called the target field, and the opposite side was called non-tarhget field. Detailed procedures for preparation of subject animals, habituation, social targets, sociability and social interaction tests, and handling of the behavior test room were carried out as described in a previous report 68.

\section{U-shaped two-choice field sociability test}

The U-shaped two-choice field sociability test was conducted as described previously ${ }^{38,39,68}$. Briefly, the Ushaped two-choice field consisted of an open field $(45 \mathrm{~cm} \times 45 \mathrm{~cm}$ ) partially partitioned with a wall (20 $\mathrm{cm}$ in width $\times 35 \mathrm{~cm}$ in height) to the central point to produce two symmetrical rectangular fields. A circular grid cage $(12 \mathrm{~cm}$ in diameter $\times 33 \mathrm{~cm}$ height) was placed on each side of the wall. For habituation, a subject mouse was allowed to freely explore the U-shaped field containing an empty grid cage on each side for $5 \mathrm{~min}$. While the subject mouse was returned to its home cage for $2 \mathrm{~min}$, a social target was loaded into one of the grid cages. Afterward, the subject mouse was again allowed to explore the U-shaped field for $5 \mathrm{~min}$, and the time spent and trajectory taken were recorded. The side containing the social target was called the target field, and the opposite side was called non-target field.

\section{Open-field social interaction test}

The open field version of the social interaction test was carried out as described previously ${ }^{59,60}$. Briefly, a mouse was placed in an open field $(45 \mathrm{~cm} \times 45 \mathrm{~cm} \times 40 \mathrm{~cm})$ containing an empty perforated interaction box at one side and allowed to explore it for $2.5 \mathrm{~min}$ for habituation. While the subject mouse was returned to its home cage for $1 \mathrm{~min}$, a social target was placed in the interaction box, and then the subject mouse was allowed to freely explore the open field for $2.5 \mathrm{~min}$. The time spent interacting with the social target and locomotive activity in the field were recorded. 


\section{Sucrose preference test}

The sucrose preference test (SPT) was carried out as described previously ${ }^{69}$. Briefly, mice were habituated to two water bottles for seven days, followed by the presentation of two bottles containing a $1 \%$ sucrose solution for $24 \mathrm{~h}$. The mice were then deprived of water and sucrose solution for $9 \mathrm{~h}$ (10 AM $7 \mathrm{PM}$ ). Beginning at $5 \mathrm{PM}$, the mice were singly housed in a cage provided with food but no water. At 7 PM, each mouse was given two bottles, one containing water and the other containing a $1 \%$ sucrose solution. The positions of the water and sucrose bottles were changed at 8 PM, 9 PM, and 10 PM. The amounts of water and sucrose consumed were measured by weighing the bottles. The sucrose preference (\%) was calculated as the amount of sucrose solution consumed over the amount of water for $2 \mathrm{~h}(8 \mathrm{PM}-10 \mathrm{PM})$.

\section{Tail suspension test}

The tail suspension test was carried out as described previously ${ }^{38,39}$. Briefly, mice were individually suspended for 6 min by fixing their tails with adhesive tape to the ceiling of a rectangular box, leaving them $50 \mathrm{~cm}$ above the table surface. During the 6 min period, the cumulative immobility time was counted. Immobility was defined as the total time in which all limbs and the body did not move.

\section{Forced swim test}

The forced swim test was carried out as described previously ${ }^{38,39}$. Briefly, mice were placed for 6 min in a transparent Plexiglass cylinder (15 cm in diameter x $27 \mathrm{~cm}$ in height) containing water (a depth of $15 \mathrm{~cm}$ ) at $24{ }^{\circ} \mathrm{C}$. The cumulative immobility time was counted for the last $5 \mathrm{~min}$. Immobility was defined as floating with all limbs motionless.

\section{Principal component analysis and $K$-Means clustering}

The behavioral features of individual animals in multiple behavior tests were analyzed using $K$-Means clustering, an unsupervised machine learning algorithm that can portion individual data points into distinct subgroups without reference to known or labeled outcomes ${ }^{70}$. The principal component analysis (PCA) was used to transform the behavioral performance of individual animals in two behavior tests into a single eigenvector in a linear dimension, under the following conditions: $p<0.05$ of Bartlett's test of sphericity, $\geq 0.5$ of the Kaiser-Meyer-Olkin measure of sampling adequacy, $\geq 0.5$ of communality, and $\geq 1.0$ of the eigenvalues in the correlation matrix. The proportion of variance explained (PVE) for the dimension reductions in the present study was in the range of $73.4 \%-86.3 \%$. All individual variables in 
the behavioral tests and all transformed values were normalized into z-scores by calculating the mean ( $\mu$ ) and standard deviation $(\sigma)$. The z-scores were calculated as given below.

$z=(x-\mu) / \sigma$

$K$-Means clustering of the behavioral features of individual animals measured by 3 behavior tests were presented in 3-dimensional matrices. The behavioral features of individual animals in 4 - 5 behavior tests and other physiological measurements of individual animals were visualized in a 3-dimensional matrix for convenience after dimensional reduction of the TST x FST or SIT x SPT components. Prepared individual data points were randomly selected to find two centroids by computing the sum of the squared distance between data points. The two centroids used the following objective function $(J)$ to subsequently assign each data point to the closest cluster center.

The objective function is:

$J=\sum_{j=1}^{2} \sum_{i=1}^{n}\left\|x_{i}^{(j)}-c_{j}\right\|^{2}$

$J=$ objective function, $k=2$ (number of clusters), $n=$ number of subject groups, $x_{i}=$ values from behavioral tests, $c_{j}=$ centroid value for cluster.

Finally, the proportion (\%) of partitioned data points in the two clusters was evaluated for each experimental group. PCA and K-Means clustering were carried out using SPSS 25.0 software (IBM, Armonk, NY, USA).

\section{Statistical analysis}

Statistical analyses were conducted using GraphPad Prism 6 software (GraphPad Software, Inc., CA, USA). Two-sample comparisons were performed using Student's t-test, and multiple comparisons were performed using one-way ANOVA and the Newman-Keuls post-hoc test. All data are presented as mean \pm SEM, and statistical differences were accepted at $p<0.05$ unless otherwise indicated. The statistical details of the results of all figures are provided in Supplementary Table 4.

\section{Data availability}


Further information and requests for resources and reagents should be directed to and will be fulfilled by the corresponding author upon request.

\section{Declarations}

\section{Competing interests}

The authors have no competing financial interests to declare.

\section{Author Contributions}

E.-H.L, J.-Y.P., and H.-J.K. carried out the experiments; E.-H.L. and P.-L.H. designed the experiments, performed the statistical analysis, and wrote the manuscript.

\section{Acknowledgments}

This research was supported by a grant (2018R1A2B2001535) from the Ministry of Science, ICT and Future Planning, Republic of Korea.

\section{References}

1. Beck, A.T. Cognitive Therapy: Nature and Relation to Behavior Therapy - Republished Article. Behav. Ther. 47, 776-784 (2016).

2. Gross, J.J. \& John, O.P. Individual differences in two emotion regulation processes: implications for affect, relationships, and well-being. J. Pers. Soc. Psychol. 85, 348-362 (2003).

3. Gross, J.J. Emotion regulation: taking stock and moving forward. Emotion 13, 359-365 (2013).

4. Forkmann, T. et al. Does cognitive behavior therapy alter emotion regulation in inpatients with a depressive disorder? Psychol. Res. Behav. Manag. 7, 147-153 (2014).

5. Goldin, P.R. et al. Impact of cognitive behavioral therapy for social anxiety disorder on the neural dynamics of cognitive reappraisal of negative self-beliefs: randomized clinical trial. JAMA Psychiatry 70, 1048-1056 (2013).

6. Kaczkurkin, A.N. \& Foa, E.B. Cognitive-behavioral therapy for anxiety disorders: an update on the empirical evidence. Dialogues Clin. Neurosci. 17, 337-346 (2015).

7. Hofmann, S.G. \& Smits, J.A. Cognitive-behavioral therapy for adult anxiety disorders: a meta-analysis of randomized placebo-controlled trials. J. Clin. Psychiatry 69, 621-632 (2008).

8. Disner, S.G., Beevers, C.G., Haigh, E.A. \& Beck, A.T. Neural mechanisms of the cognitive model of depression. Nat. Rev. Neurosci. 12, 467-477 (2011). 
9. Grosse Holtforth, M. et al. A randomized-controlled trial of cognitive-behavioral therapy for depression with integrated techniques from emotion-focused and exposure therapies. Psychother. Res. 29, 30-44 (2019).

10. McEwen, B.S. Stress, adaptation, and disease. Allostasis and allostatic load. Ann. NY Acad. Sci. 840, 33-44 (1998).

11. Nestler, E.J. et al. Neurobiology of depression. Neuron 34, 13-25 (2002).

12. Selye, H. The stress of life. New York: McGraw-Hill (1956).

13. Selye, H. A Syndrome produced by Diverse Nocuous Agents. Nature 138, 32 (1936).

14. Young, E.A., Abelson, J. \& Lightman, S.L. Cortisol pulsatility and its role in stress regulation and health. Front. Neuroendocrinol. 25, 69-76 (2004).

15. de Kloet, E.R., Joels, M. \& Holsboer, F. Stress and the brain: from adaptation to disease. Nat. Rev. Neurosci. 6, 463-475 (2005).

16. Herman, J.P., McKlveen, J.M. \& Ghosal, S. Regulation of the Hypothalamic-Pituitary-Adrenocortical Stress Response. Compr. Physiol. 6, 603-621 (2016).

17. Erickson, K., Drevets, W. \& Schulkin, J. Glucocorticoid regulation of diverse cognitive functions in normal and pathological emotional states. Neurosci. Biobehav. Rev. 27, 233-246 (2003).

18. Myers, B., McKlveen, J.M. \& Herman, J.P. Glucocorticoid actions on synapses, circuits, and behavior: Implications for the energetics of stress. Front Neuroendocrinol. 35, 180-196 (2014).

19. McEwen, B.S., Nasca, C. \& Gray, J.D. Stress Effects on Neuronal Structure: Hippocampus, Amygdala, and Prefrontal Cortex. Neuropsychopharmacology 41, 3-23 (2016).

20. Arnsten, A.F. Stress signalling pathways that impair prefrontal cortex structure and function. Nat. Rev. Neurosci. 10, 410-422 (2009).

21. Pittenger, C. \& Duman, R.S. Stress, depression, and neuroplasticity: a convergence of mechanisms. Neuropsychopharmacology 33, 88-109 (2008).

22. Popoli, M., Yan, Z., McEwen, B.S. \& Sanacora, G. The stressed synapse: the impact of stress and glucocorticoids on glutamate transmission. Nat. Rev. Neurosci. 13, 22-37 (2011).

23. Yuen, E.Y. et al. Repeated stress causes cognitive impairment by suppressing glutamate receptor expression and function in prefrontal cortex. Neuron 73, 962-977 (2012).

24. Radley, J.J. et al. Repeated stress induces dendritic spine loss in the rat medial prefrontal cortex. Cereb. Cortex 16, 313-320 (2006).

25. Sullivan, R.M. \& Gratton, A. Prefrontal cortical regulation of hypothalamic-pituitary-adrenal function in the rat and implications for psychopathology: side matters. Psychoneuroendocrinology 27, 99114 (2002).

26. Dinan, T.G. et al. Dexamethasone augmentation in treatment-resistant depression. Acta. Psychiatr. Scand. 95, 58-61 (1997).

27. DeBattista, C., Posener, J.A., Kalehzan, B.M. \& Schatzberg, A.F. Acute antidepressant effects of intravenous hydrocortisone and $\mathrm{CRH}$ in depressed patients: a double-blind, placebo-controlled study. 
Am. J. Psychiatry 157, 1334-1337 (2000).

28. Goodwin, G.M. et al. The effects of cortisol infusion upon hormone secretion from the anterior pituitary and subjective mood in depressive illness and in controls. J. Affect. Disord. 26, 73-83 (1992).

29. Stone, E.A. \& Lin, Y. An anti-immobility effect of exogenous corticosterone in mice. Eur. J. Pharmacol. 580, 135-142 (2008).

30. Pariante, C.M., The glucocorticoid receptor: part of the solution or part of the problem? J. Psychopharmacol. 20, 79-84 (2006).

31. Szklarczyk, D. et al. STRING v11: protein-protein association networks with increased coverage, supporting functional discovery in genome-wide experimental datasets. Nucleic Acids Res. 47, D607D613 (2019).

32. Galigniana N.M. et al. Regulation of the glucocorticoid response to stress-related disorders by the Hsp90-binding immunophilin FKBP51. J. Neurochem. 122, 4-18 (2012).

33. Wochnik, G.M. et al. FK506-binding proteins 51 and 52 differentially regulate dynein interaction and nuclear translocation of the glucocorticoid receptor in mammalian cells. J. Biol. Chem. 280, 46094616 (2005).

34. Guidotti, G. et al. Glucocorticoid receptor and FKBP5 expression is altered following exposure to chronic stress: modulation by antidepressant treatment. Neuropsychopharmacology 38, 616-627 (2013).

35. Lee, Y.A. \& Goto, Y. Chronic stress modulation of prefrontal cortical NMDA receptor expression disrupts limbic structure-prefrontal cortex interaction. Eur. J. Neurosci. 34, 426-436 (2011).

36. Bali, B., Erdélyi, F., Szabó, G. \& Kovács, K.J. Visualization of stress-responsive inhibitory circuits in the GAD65-eGFP transgenic mice. Neurosci. Lett. 380, 60-65 (2005).

37. Radley, J.J., Gosselink, K.L. \& Sawchenko, P.E. A discrete GABAergic relay mediates medial prefrontal cortical inhibition of the neuroendocrine stress response. J. Neurosci. 29, 7330-7340 (2009).

38. Kim, T.K. et al. G9a-Mediated Regulation of OXT and AVP Expression in the Basolateral Amygdala Mediates Stress-Induced Lasting Behavioral Depression and Its Reversal by Exercise. Mol. Neurobiol. 53, 2843-2856 (2016).

39. Seo, J.S. et al. NADPH oxidase mediates depressive behavior induced by chronic stress in mice. J. Neurosci. 32, 9690-9699 (2012).

40. Carroll, B.J. et al. Pathophysiology of hypercortisolism in depression. Acta. Psychiatr. Scand. Suppl. 433, 90-103 (2007).

41. Parker, K.J., Schatzberg, A.F. \& Lyons, D.M. Neuroendocrine aspects of hypercortisolism in major depression. Horm. Behav. 43, 60-66 (2003).

42. Belanoff, J.K., Flores, B.H., Kalezhan, M., Sund, B. \& Schatzberg, A.F. Rapid reversal of psychotic depression using mifepristone. J. Clin. Psychopharmacol. 21, 516-521 (2001). 
43. David, D.J. et al. Neurogenesis-dependent and -independent effects of fluoxetine in an animal model of anxiety/depression. Neuron 62, 479-493 (2009).

44. Johnson, S.A., Fournier, N.M. \& Kalynchuk, L.E. Effect of different doses of corticosterone on depression-like behavior and HPA axis responses to a novel stressor. Behav. Brain Res. 168, 280-288 (2006).

45. Kalafatakis, $\mathrm{K}$ et al. Ultradian rhythmicity of plasma cortisol is necessary for normal emotional and cognitive responses in man. Proc. Natl. Acad. Sci. 115, E4091-E4100 (2018).

46. Lightman, S.L. \& Conway-Campbell, B.L. The crucial role of pulsatile activity of the HPA axis for continuous dynamic equilibration. Nat. Rev. Neurosci. 11, 710-718 (2010).

47. Fries, G.R., Gassen, N.C. \& Rein, T. The FKBP51 Glucocorticoid Receptor Co-Chaperone: Regulation, Function, and Implications in Health and Disease. Int. J. Mol. Sci. 18, 2614 (2017).

48. Jett, J.D., Bulin, S.E., Hatherall, L.C., McCartney, C.M. \& Morilak, D.A. Deficits in cognitive flexibility induced by chronic unpredictable stress are associated with impaired glutamate neurotransmission in the rat medial prefrontal cortex. Neuroscience 346, 284-297 (2017).

49. Son, $\mathrm{H}$. et al. Glutamine has antidepressive effects through increments of glutamate and glutamine levels and glutamatergic activity in the medial prefrontal cortex. Neuropharmacology 143, 143-152 (2018).

50. Veeraiah, P. et al. Dysfunctional glutamatergic and $y$-aminobutyric acidergic activities in prefrontal cortex of mice in social defeat model of depression. Biol. Psychiatry 76, 231-238 (2014).

51. Covington, H.E. et al. Antidepressant effect of optogenetic stimulation of the medial prefrontal cortex. J. Neurosci. 30, 16082-16090 (2010).

52. Bagot, R.C. et al. Ventral hippocampal afferents to the nucleus accumbens regulate susceptibility to depression. Nat. Commun. 6, 7626 (2015).

53. Wei, J., Zhong, P., Qin, L., Tan, T. \& Yan, Z. Chemicogenetic Restoration of the Prefrontal Cortex to Amygdala Pathway Ameliorates Stress-Induced Deficits. Cereb. Cortex 28, 1980-1990 (2018).

54. Ashokan, A., Hegde, A. \& Mitra, R. Short-term environmental enrichment is sufficient to counter stressinduced anxiety and associated structural and molecular plasticity in basolateral amygdala. Psychoneuroendocrinology 69, 189-196 (2016).

55. Brockhurst, J., Cheleuitte-Nieves, C., Buckmaster, C.L., Schatzberg, A.F. \& Lyons, D.M. Stress inoculation modeled in mice. Transl. Psychiatry 5, e537 (2015).

56. Parihar, V.K., Hattiangady, B., Kuruba, R., Shuai, B. \& Shetty, A.K. Predictable chronic mild stress improves mood, hippocampal neurogenesis and memory. Mol. Psychiatry 16, 171-183 (2011).

57. Suo, L. et al. Predictable chronic mild stress in adolescence increases resilience in adulthood. Neuropsychopharmacology 38, 1387-1400 (2013).

58. Kim, K.S. \& Han, P.L. Optimization of chronic stress paradigms using anxiety- and depression-like behavioral parameters. J. Neurosci. Res. 83, 497-507 (2006). 
59. Berton, O. et al. Essential role of BDNF in the mesolimbic dopamine pathway in social defeat stress. Science 311, 864-868 (2006).

60. Golden, S.A., Covington, H.E. 3rd, Berton, O. \& Russo, S.J. A standardized protocol for repeated social defeat stress in mice. Nat. Protoc. 6, 1183-1191 (2011).

61. Kim, K.S. \& Han, P.L. Mice lacking adenylyl cyclase-5 cope badly with repeated restraint stress. J. Neurosci. Res. 87, 2983-2993 (2009).

62. Hultman, R. et al. Dysregulation of Prefrontal Cortex-Mediated Slow-Evolving Limbic Dynamics Drives Stress-Induced Emotional Pathology. Neuron 91, 439-452 (2016).

63. Kim, H. et al. Adenylyl cyclase-5 in the dorsal striatum function as a molecular switch for the generation of behavioral preferences for cue-directed food choices. Mol. Brain 7, 77 (2014).

64. Lehmann, M.L., Brachman, R.A., Martinowich, K., Schloesser, R.J. \& Herkenham, M. Glucocorticoids orchestrate divergent effects on mood through adult neurogenesis. J. Neurosci. 33, 2961-2972 (2013).

65. Bult, C.J., Blake, J.A., Smith, C.L., Kadin, J.A. \& Richardson, J.E. Mouse Genome Database Group. Mouse Genome Database (MGD) 2019. Nucleic Acids Res. 47, D801-D806 (2019).

66. Doncheva, N.T., Morris, J.H., Gorodkin, J. \& Jensen, L.J. Cytoscape StringApp: Network Analysis and Visualization of Proteomics Data. J. Proteome Res. 18, 623-632 (2019).

67. Nadler, J.J. et al. Automated apparatus for quantitation of social approach behaviors in mice. Genes Brain Behav. 3, 303-314 (2004).

68. Lee, E., Park, J., Lee, Y. \& Han, P. Sociability and Social Novelty Preference Tests Using a U-shaped Two-choice Field. Bio-protocol 8, e2853 (2018).

69. Iñiguez, S.D. et al. Extracellular signal-regulated kinase-2 within the ventral tegmental area regulates responses to stress. J. Neurosci. 30, 7652-7663 (2010).

70. Jain, A.K. Data clustering: 50 years beyond k-means. Pattern Recognition Letters 31, 651-666 (2010).

\section{Figures}




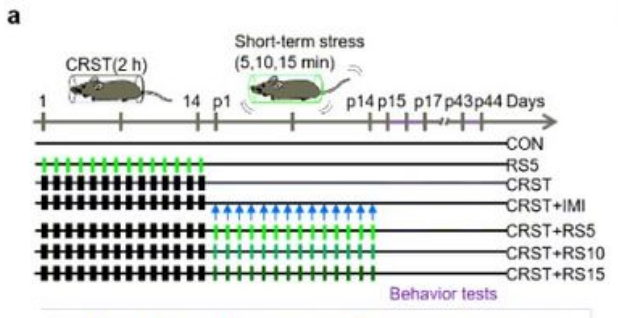

I $2 \mathrm{~h} 15 \mathrm{~min} / 10 \mathrm{~min} / 15 \mathrm{~min}$ restraint $\uparrow$ Imipramine (20 mg/kg, i.p.)
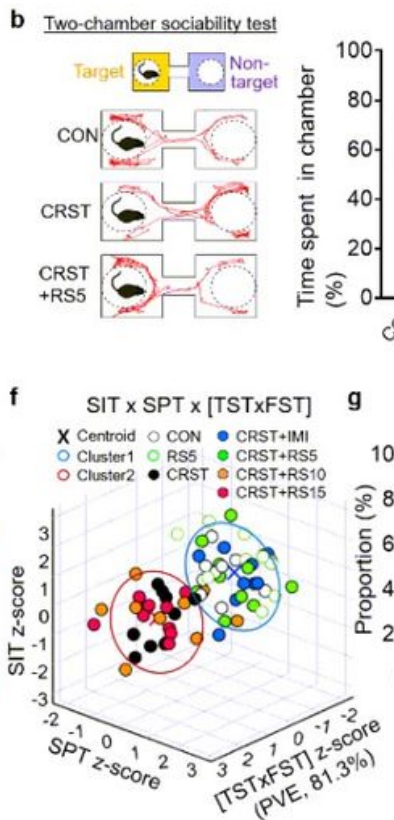
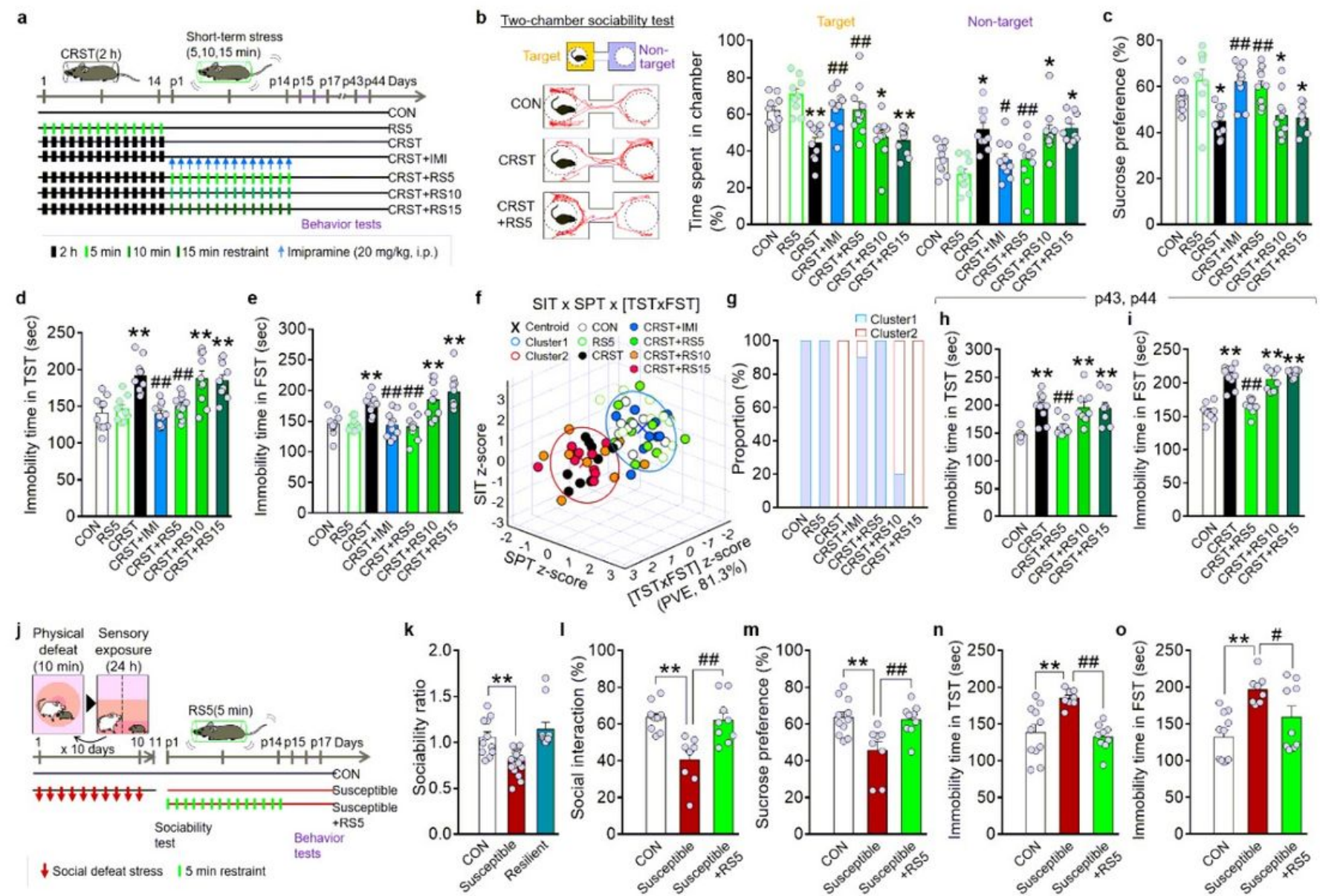

\section{Figure 1}

Repeated treatment with a short sequence of behavioral stress produces anti-depressive effects in stressinduced models of depression. a, Experimental design. Mice were treated with 5-min or 2-h restraint for 14 days. CRST mice were treated with 5-min, 10-min, or 15-min restraint or imipramine for 14 days (RS5, RS10, RS15, and IMI, respectively), and then given behavioral tests. b-g, Representative tracks and \% time spent in the target and non-target chambers in the SIT (b), sucrose preference in the SPT (c), and immobility time in the TST (d) and FST (e) for the indicated groups on post-stress days 15-17. K-Means clustering ( $k=2)$ of individuals in the SIT x SPT x [TST x FST] matrix ( $f)$ and \% composition of each group in the clusters $(\mathrm{g})$. PCA was used for dimension reduction of the TST $x$ FST components (PVE, 81.3\%) (n = 9-10 per group). $\mathrm{h}-\mathrm{I}$, Immobility time in the TST (h) and FST (i) for the indicated groups on post-stress days 43 and 44 ( $n=8-10$ per group). j, Experimental design. The susceptible mice were treated with RS5, and then given behavioral tests. $\mathrm{k}-\mathrm{o}$, Mice susceptible or resilient to CSDS were separated by the sociability ratio (k). \% time spent in the target chamber in the two-chamber SIT (I), sucrose preference in the SPT $(m)$, and immobility time in the TST $(n)$ and FST (o) for the indicated groups ( $n=8-11$ per group). Data are mean \pm SEM. *, difference compared to control; \#, difference compared to CRST. *, \#, $p<0.05$; **, $\# \#, p<0.01$ (One-way ANOVA followed by Newman-Keuls post-hoc test). See Supplementary Table 4 for statistical details. 

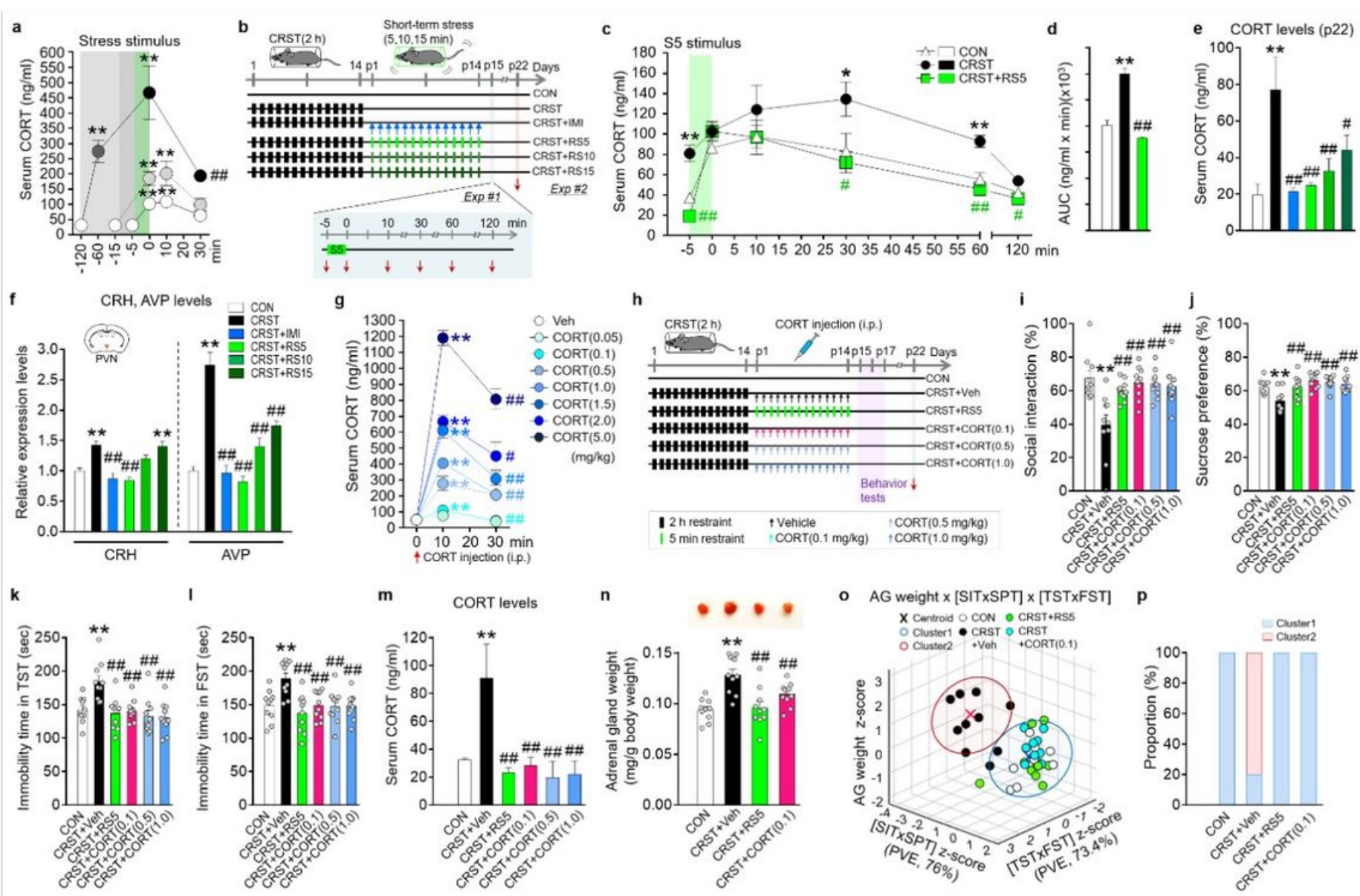

Figure 2

RS5 and low-dose CORT normalize stress-induced dysregulation of the HPA axis. a, Serum CORT levels in mice treated with 5-min, 15-min, 60-min, and 120-min restraint ( $n=8-9$ per group). $b-f$, Experimental design (b). Time course of serum CORT levels in the indicated groups after exposure to a single 5-min restraint (S5) (Exp \#1) (c), and the area under the curve (AUC) between -5 min and 120 min (d) ( $n=7-8$ per group). Basal serum CORT levels (e) and CRH and AVP expression levels in the PVN (f) of the indicated groups (Exp \#2) ( $n=7-8$ per group). g, Changes in serum CORT levels in mice after CORT injection ( $n=8$ 9 per group). $\mathrm{h}-\mathrm{p}$, Experimental design (h). CORT was injected for 14 days $(0.1,0.5,1.0 \mathrm{mg} / \mathrm{kg} / \mathrm{day}$, i.p.) in CRST mice. Behavioral performance in the SIT (i), SPT (j), TST (k), and FST (I) for the indicated groups ( $n=10$ per group). Basal serum CORT levels ( $n=8$ per group) $(m)$ and adrenal gland (AG) weight $(n=10$ per group) (n) of the indicated groups on post-stress day 22. K-Means clustering of individuals in the [AG weight] $x$ [SIT x SPT] x [TST x FST] matrix (o) and \% composition of each group in the clusters (p). PCA was used for dimensional reduction of the SIT and SPT components (PVE; 76.0\%) and the TST and FST components (PVE; 73.4\%). Data are mean \pm SEM. *, difference compared to control; \#, difference compared to CRST. *, \#, p < 0.05; **, \#\#, p < 0.01 (One-way ANOVA followed by Newman-Keuls post-hoc test). See Supplementary Table 4 for statistical details. 

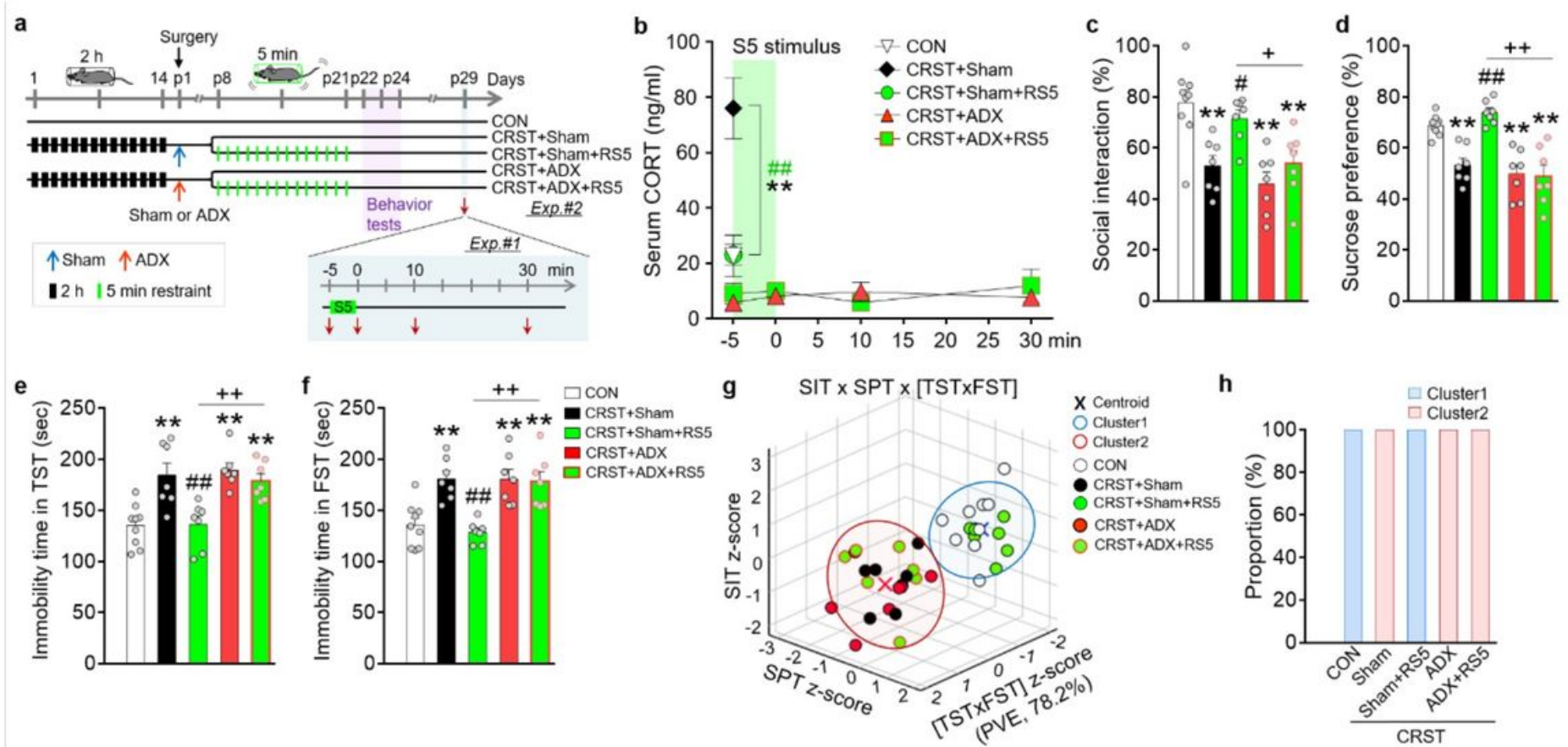

\section{Figure 3}

Adrenalectomy (ADX) blocks the antidepressive effects of RS5 in CRST mice. a, Experimental design for treatment with CRST, ADX or sham surgery, followed by RS5 treatment and behavioral tests. Red arrows $(\downarrow)$ in Exp \#1, Serum collection point. Mice were sacrificed after exposure to a single 5-min restraint (S5). $b-h$, The basal CORT levels at $-5 \mathrm{~min}$, and the time course of serum CORT levels in the indicated groups after exposure to S5 (Exp \#1) (b) ( $n=7-10$ per group). Social interaction in the SIT (c), sucrose preference in the SPT (d), and immobility time in the TST (e) and FST (f) for the indicated groups (Exp \#2). K-Means clustering of individuals in the SIT x SPT x [TST x FST] matrix (g) and \% composition of each group in the clusters (h). The TST and FST components were transformed into linear eigenvectors using PCA (PVE; $78.2 \%)(n=7-10$ per group). Data are mean \pm SEM. *, difference compared to control; \#, difference compared to CRST; +, difference compared to CRST+RS5. ${ }^{*}, \#,+, p<0.05 ; * \star$, \#\#,,$++ p<0.01$ (One-way ANOVA followed by Newman-Keuls post-hoc test). See Supplementary Table 4 for statistical details. 

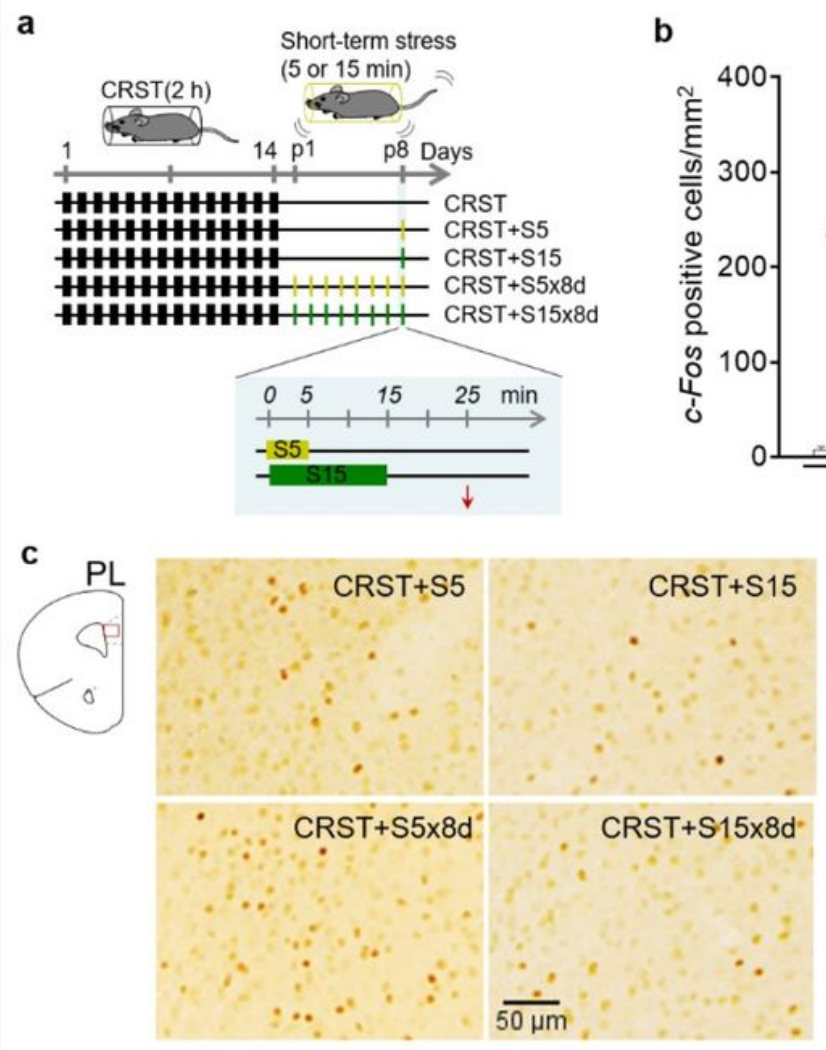
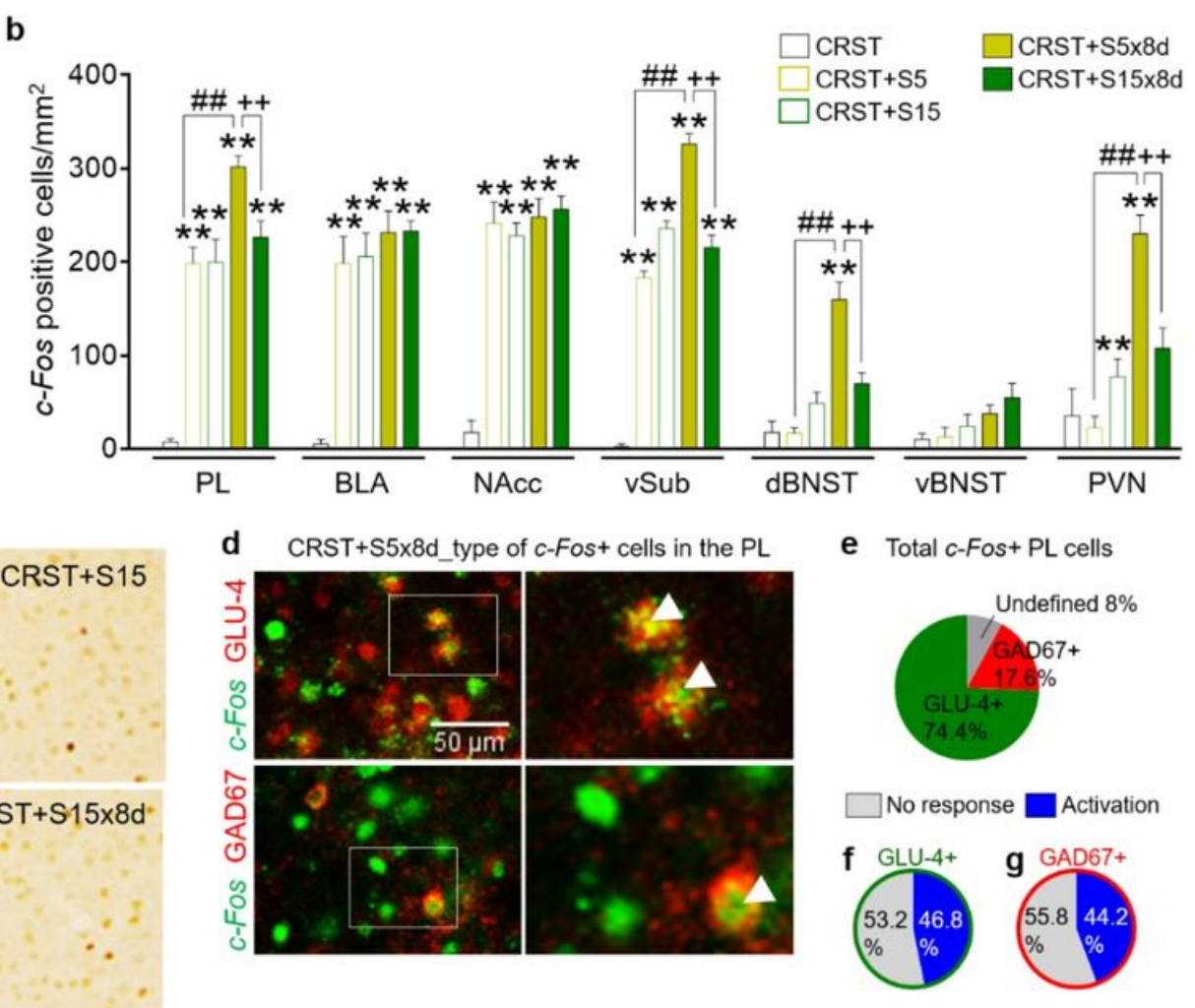

\section{Figure 4}

Repeated treatment with a short sequence of behavioral stress recruits the brain regions regulating stress coping in CRST mice. a, Experimental design. CRST mice were treated with a single 5-min or 15-min restraint (S5 and S15, respectively) on post-stress day 8, and then sacrificed, 20 min and 10 min later, respectively. Another group of CRST mice was treated with 5-min or 15-min restraint for 8 days (S5x8d and S15x8d, respectively), and then sacrificed, 20 min and 10 min, respectively, after the last restraint. b,c, Quantification of c-Fos expression levels in the PL, BLA, NAcc, vSub, dBNST, ventral BNST (vBNST), and PVN (b). Photomicrographs showing c-Fos expression in the PL (red box) (C) of the S5, S15, S5x8d, and S15x8d groups ( $n=4-6$ per group). $d-g$, Photomicrographs showing c-Fos induction in GLU-4-positive or GAD67-positive neurons in the PL (d), and their quantification levels (e-g) ( $n=6$ animals for each). The details are shown in Supplementary Table 1. Data are mean \pm SEM. *, **, difference compared to control; $\#$, difference compared to CRST; + , difference between indicated groups. $* *, \# \#,++, p<0.01$ (one-way ANOVA followed by Newman-Keuls post-hoc test). See Supplementary Table 4 for statistical details. 


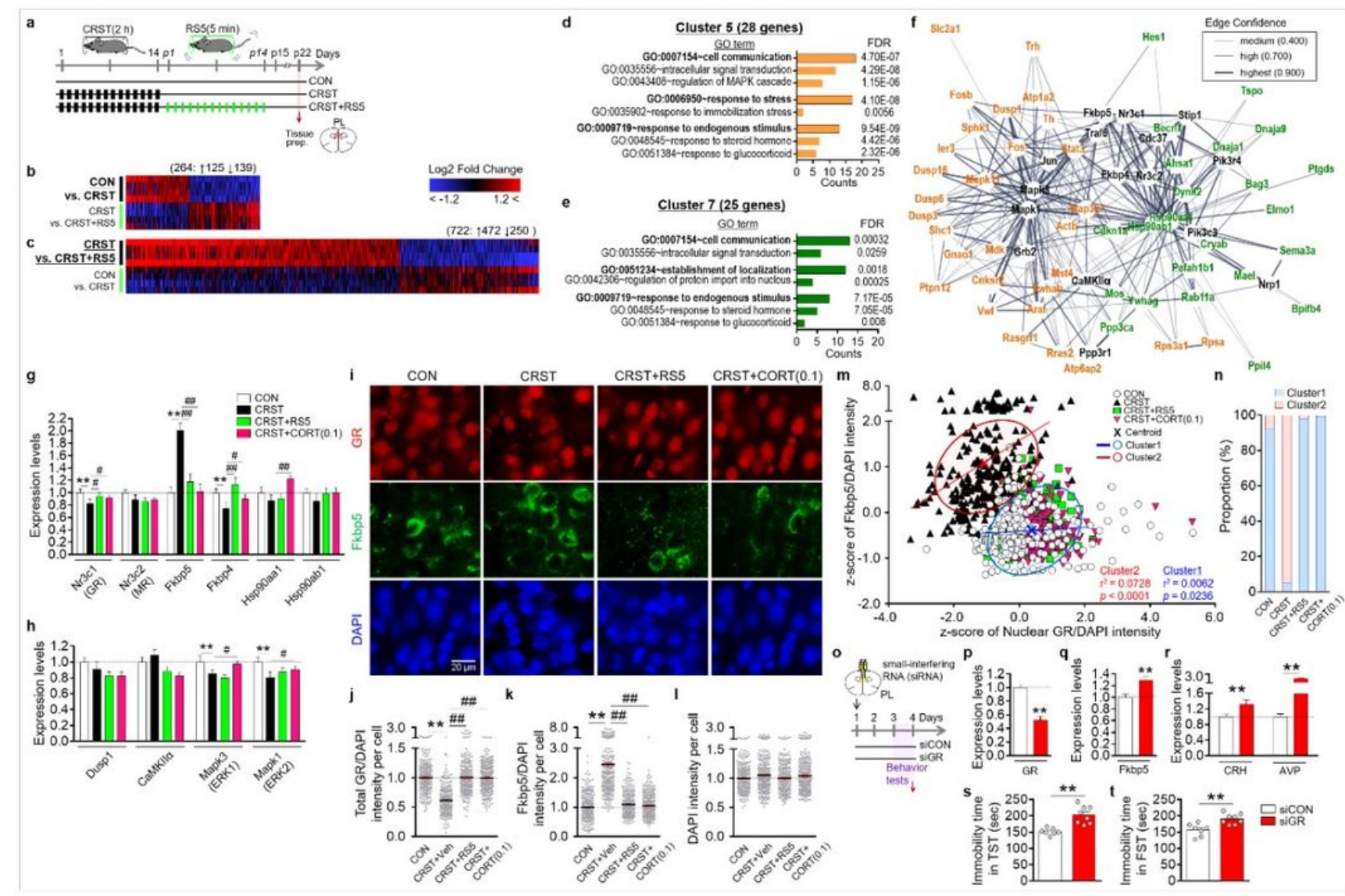

\section{Figure 5}

RS5 treatment reverses stress-induced changes in gene expression profiles in the PL. a-c, Experimental design (a). Heatmap showing the expression profiles were changed by $\geq 1.2$-fold after CRST (b) and reversed by RS5 treatment in CRST mice (c), and follow-up unbiased alignment with the expression levels of the respective genes changed by RS5 (b) and CRST (c). $\uparrow$ and $\downarrow$, indicate up- and down-regulation, respectively. $d-f, G O$ enrichment analysis with weighted K-Means clustering identified the clusters which carry the functional modules labeled with the GO terms "response to stress" or "response to glucocorticoid" ( $d, e)$. The PPI networks formed by the members of clusters 5 (orange, 28 genes) and 7 (green, 24 genes) and 16 genes (black) from an extended search of the STRING database at a confidence level of >0.700 (f). g,h, Expression levels of Nr3c1, Nr3c2, Fkbp5, Fkbp4, Hsp90aa1, and Hsp90ab1 (g), and Dusp1, CaMKIla, Mapk3, and Mapk1 (h) in the PL of the indicated groups ( $\mathrm{n}=8-12$ per group). $\mathrm{i}-\mathrm{n}$, Immunofluorescence images showing GR (red) and Fkbp5 (green) expression in the PL from the indicated groups (i). DAPI, blue. Quantification levels of total GR/DAPI (j), Fkbp5/DAPI (k), and DAPI intensity (I) in individual cells ( $n=4-6$ animals per group; $n=248$ - 309 per group). Differential expression of nuclear GR and Fkbp5 in individual cells (m). Two distinct clusters with centroids classified by K-Means clustering, and the regression values $(\mathrm{m})$ and \% composition of individuals cells of each group $(\mathrm{n})$ in the clusters were indicated. o-t, Experimental design (o). siRNA-mediated knockdown of GR in the PL, and subsequent behavioral tests. Red arrow, time point for tissue prep. Transcript levels of GR (p) and Fkbp5 
(q) in the PL and of CRH and AVP (r) in the PVN. Immobility time in the TST (s) and FST (t) for each

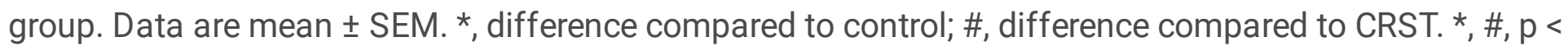
0.05; **, \#\#, p < 0.01 (One-way ANOVA followed by Newman-Keuls post-hoc test). See Supplementary Table 4 for statistical details.
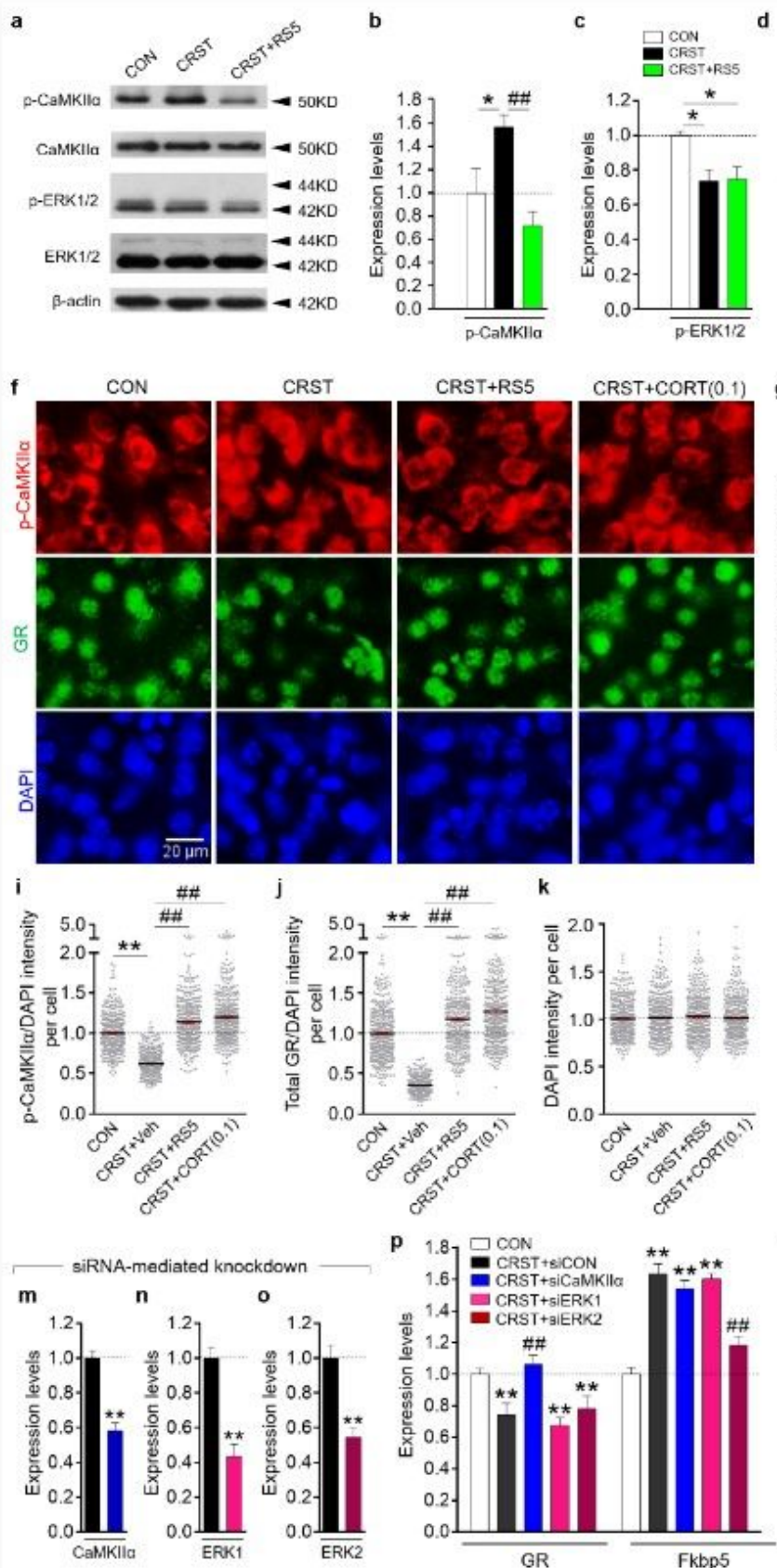
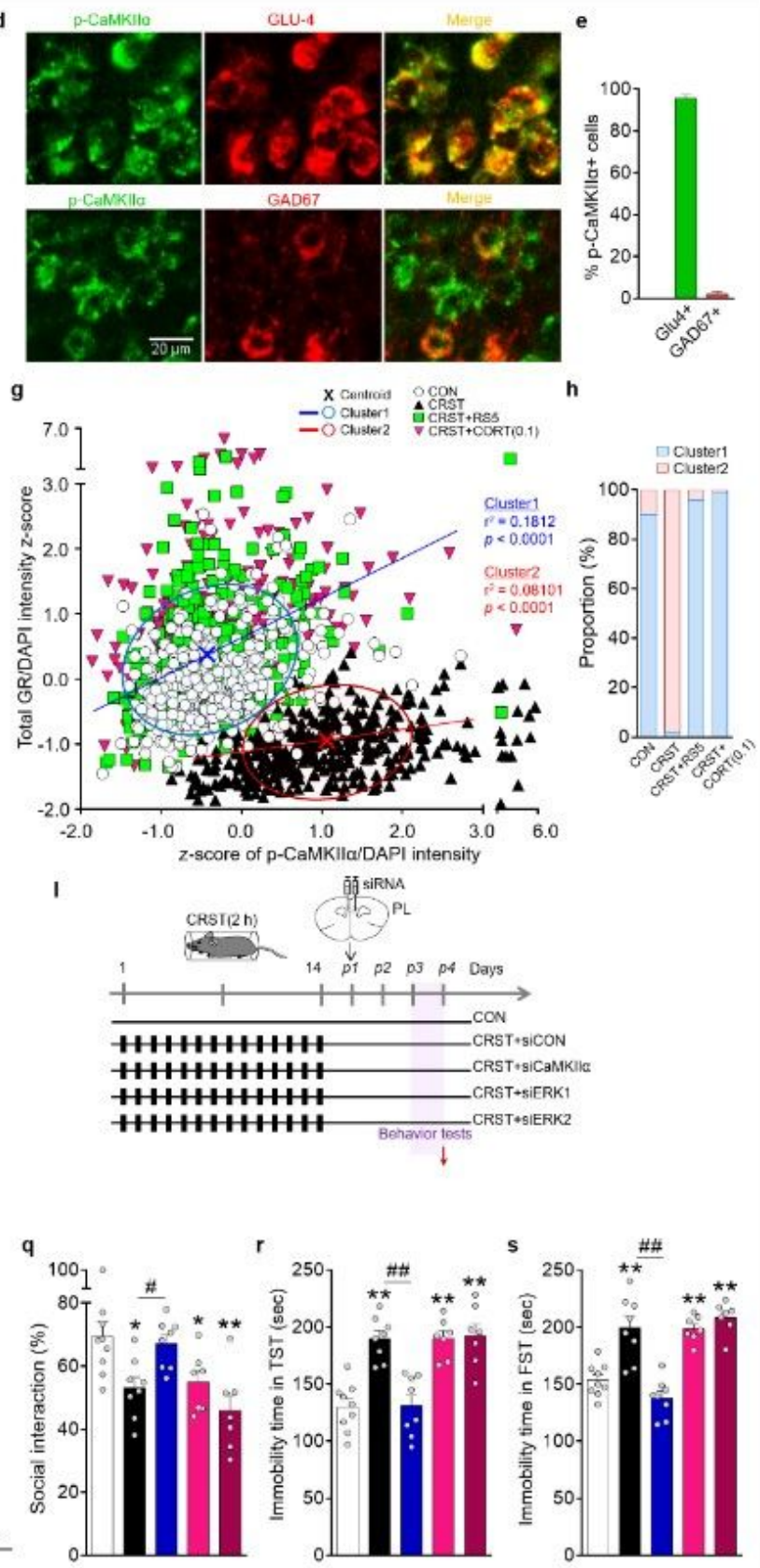

\section{Figure 6}

RS5 restores increased p-CaMKIla expression in PL neurons. a-c, Western blots showing expression levels of p-CaMKIIa, CaMKIla, p-ERK1/2, ERK1/2, and $\beta$-actin in the PL of the indicated groups (a). Quantification levels of p-CaMKIla (b) and p-ERK1/2 expression (c) ( $n=6$ animals per group, 4 repeats). d,e, Immunofluorescence staining of p-CaMKIla (green) in PL neurons stained with GLU-4 or GAD67 (red) 
(d). Quantification levels (e) ( $n=4$ per group). $f-k$, Immunofluorescence staining of $p$-CaMKIla (red) and total GR (green) in PL neurons (f). DAPI, blue. Differential expression of p-CaMKIla and total GR in individual cells of the indicated groups (g). Two distinct clusters with centroids and regression values (g) and the \% composition of individuals cells of each group in the clusters (h). Quantification levels of $p$ CaMKIla (i), GR (j) and DAPI ( $k$ ) intensity in individual cells $(n=4-6$ animals per group; $n=345-390$ per group). I-s, Experimental design (l). siRNA-mediated knockdown of CaMKIla (m), ERK1 (n), and ERK2 (o) in the PL of CRST-treated mice. Red arrow, time point for tissue prep. Transcript levels of GR and Fkbp5 $(p)$ in the PL of the indicated groups. Behavioral performance in the SIT (q), TST (r), and FST (s) for the indicated groups. Data are mean \pm SEM. *, difference compared to control; \#, difference compared to CRST. *, \#, p < 0.05, **, \#\#, p $<0.01$ (Student's t-test; One-way ANOVA followed by Newman-Keuls post-hoc test). See Supplementary Table 4 for statistical details.
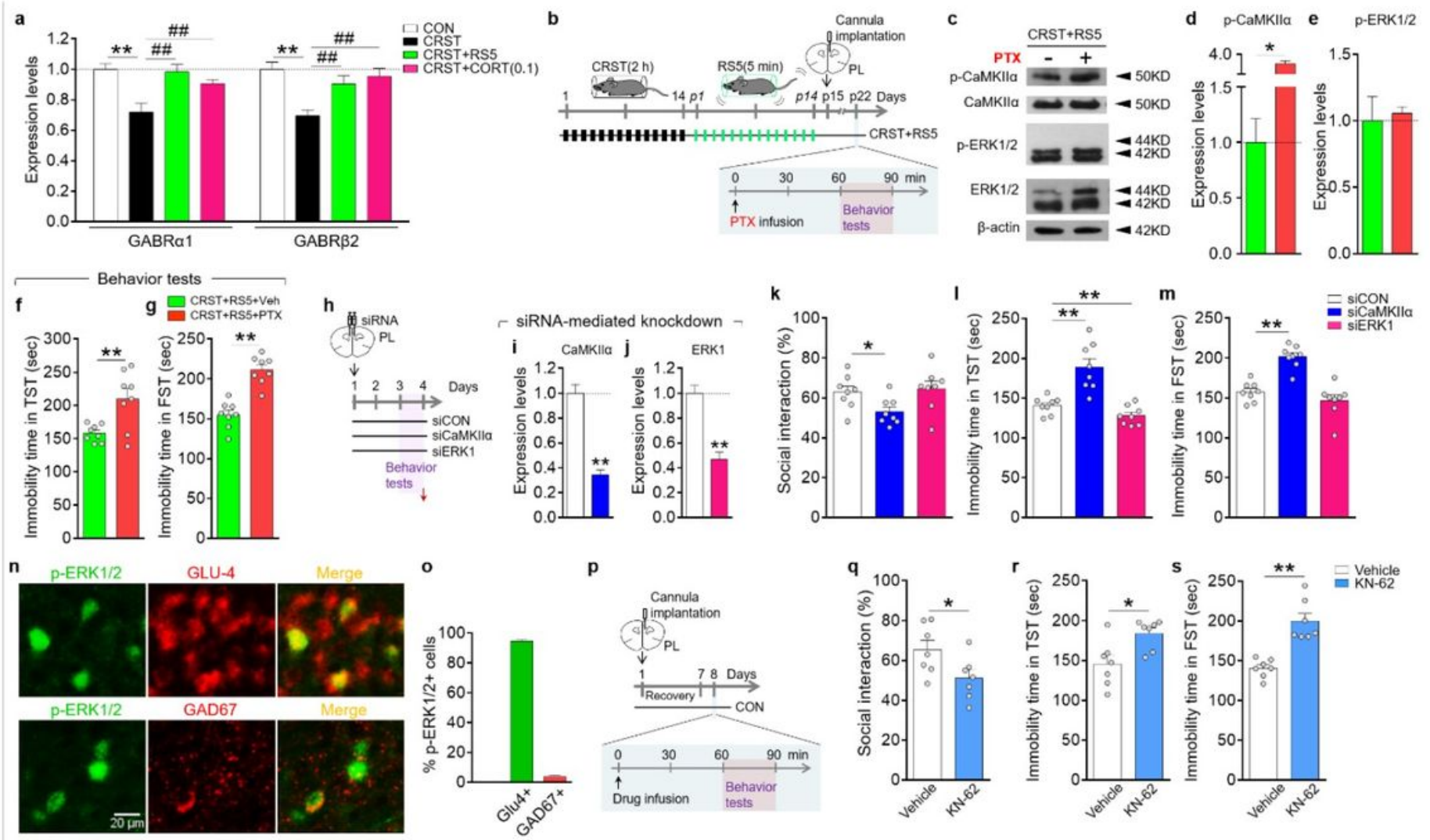

Figure 7

CaMKIla in the PL is a critical player regulating depressive behaviors. a, Transcript levels of GABRa1 and GABR 32 in the PL of the indicated groups. Sample groups were prepared from the experiments depicted in Fig. 4g,h ( $n=7-12$ per group). $b-g$, Experimental design (b). The picrotoxin (PTX) was infused using a pre-implanted cannula into the PL of mice subjected to CRST and RS5. Behavior tests were carried out between 60 and 90 min after drug infusion on post-stress day 22 (b). Western blots showing the expression levels of $p$-CaMKIla, CaMKIla, $p$-ERK1/2, ERK1/2, and $\beta$-actin in the PL of the indicated groups (c). Quantification levels of $p$-CaMKIla (d) and p-ERK1/2 (e) ( $n=6$ mice per group, 3 repeats). Behavioral performance in the TST ( $f$ ) and FST ( $g$ ) for the indicated groups ( $n=8$ per group). $h-m$, Experimental 
design (h). siRNA-mediated knockdown of CaMKIla (i) and ERK1 (j) in the PL. Behavioral tests were carried out 2 days later in the order SIT, TST, and FST. Red arrow, time point for tissue prep. Behavioral performance in the SIT $(k)$, TST $(I)$, and FST $(m)$ for the indicated groups ( $n=8$ per group). $n, 0$, Immunofluorescence staining of p-ERK1/2 (green) expression in the PL stained with GLU-4 or GAD67 (red) ( $n$ ). Quantification levels of colocalization (o) ( $n=4$ per group). $p-s$, Experimental design ( $p$ ). KN-62 (CaMKIla inhibitor, $2.5 \mathrm{nmol} /$ injection), or Veh was infused into the PL through a pre-implanted cannula. After $60 \mathrm{~min}$, behavioral tests were carried out in the order SIT, TST and FST. Behavioral performance in the SIT (q), TST (r), and FST (s) for the indicated groups ( $n=7$ per group). Data are mean \pm SEM. *, difference compared to control; \#, difference compared to CRST. *, \#, p < 0.05, **, \#\#, p 0.01 (Student's ttest; One-way ANOVA followed by Newman-Keuls post-hoc test). See Supplementary Table 4 for statistical details

a

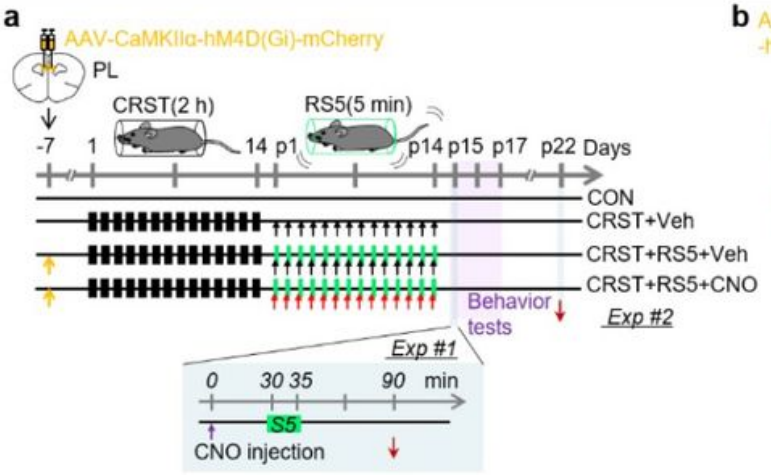

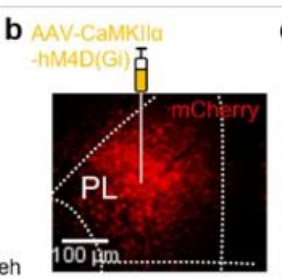

c CRST+RS5+Veh

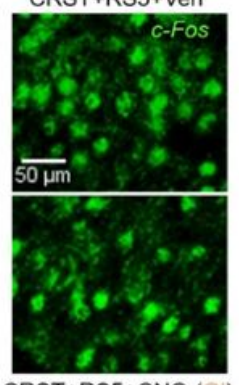

d

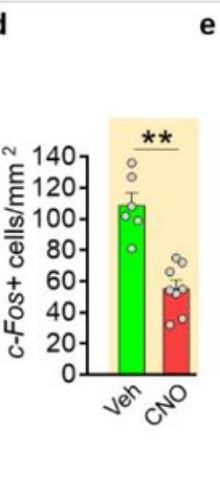

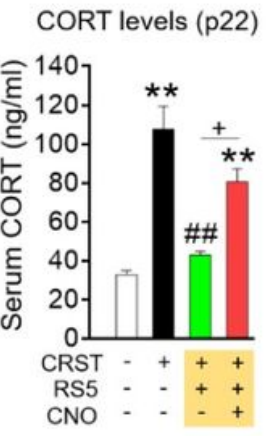

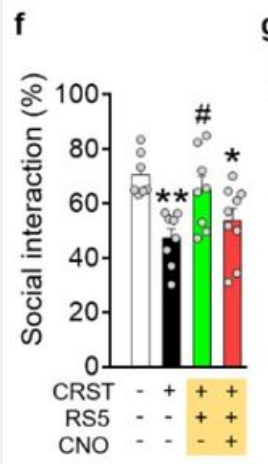

g
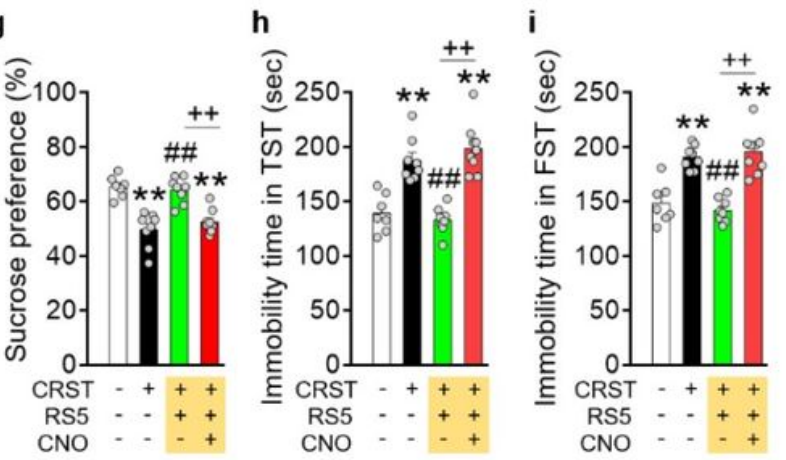

j

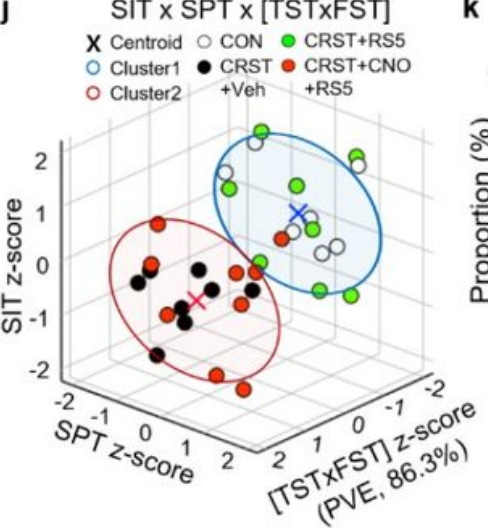

k

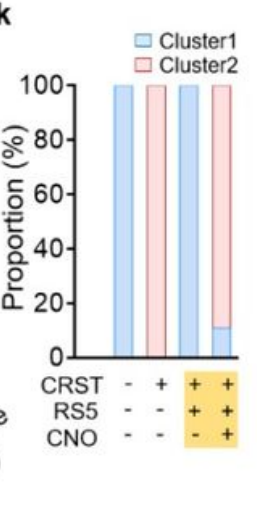

\section{Figure 8}

Chemogenetic inhibition of PL neurons blocks the anti-depressive effects of RS5. a, Experimental design. Mice injected in the PL with AAV8-CaMKIla-hM4D(Gi)-mCherry inhibitory vector were subjected to CRST, followed by RS5 treatment with Veh or CNO injection. b-e, Injection of the AAV8 vector and resulting mCherry expression in the PL (b). S5-induced c-Fos expression levels in the PL after CNO treatment (Exp \#1) (c and d) ( $n=4-6$ per group). Basal CORT levels in the indicated groups on post-stress day 22 (e) $(n=$ 7-8 per group). $f-k$, Behavioral performance in the SIT (f), SPT (g), TST (h), and FST (i) for the indicated groups (Exp \#2). K-Means clustering of individuals in the SIT x SPT x [TST x FST] matrix (J) and \% composition of each group in the clusters $(K)(n=7-9$ per group). Data are mean \pm SEM. *, difference 
compared to control; \#, difference compared to CRST. *, \#, p < 0.05, **, \#\#, p $<0.01$ (Student's t-test; oneway ANOVA followed by Newman-Keuls post-hoc test). See Supplementary Table 4 for statistical details.
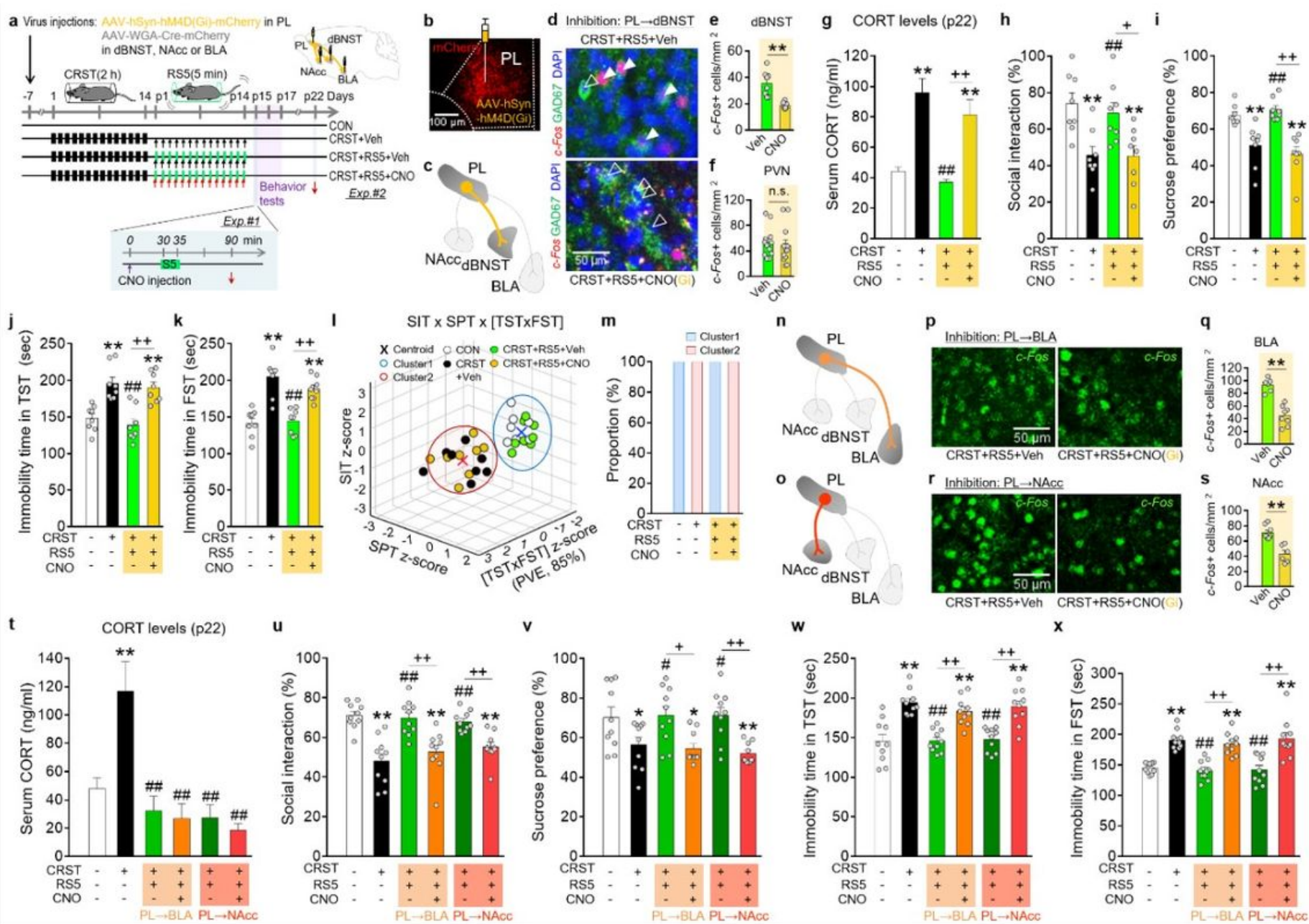

$\mathrm{x}$

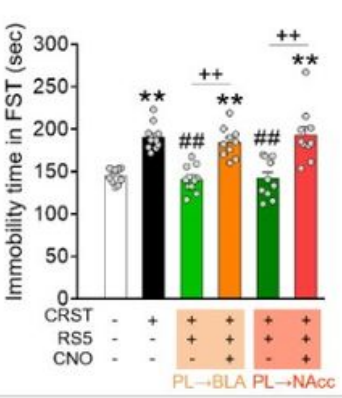

\section{Figure 9}

Chemogenetic inhibition of PL neurons projecting to the dBNST, BLA or NAcc blocks the anti-depressive effects of RS5. a, Experimental design. Mice injected in the PL with the AAV8-hSyn-hM4D(Gi)-mCherry and retrograde Cre vector in the dBNST, BLA, or NAcc were subjected to CRST, followed by RS5 treatment with Veh or CNO injection. b,c, Injection of the AAV8 vector and resulting mCherry expression in the PL (b) and the PLIdBNST circuit (c), which was labeled with hM4D(Gi) by injection of viral vectors (a). $d-f$, S5induced c-Fos expression levels in the dBNST ( $d$ and e) and PVN (f) after CNO treatment (Exp \#1) ( $n=4$ per group). c-Fos, red; GAD67, green; DAPI, blue. g, Basal CORT levels in the indicated groups on poststress day 22 ( $n=8$ per group). $h-m$, Behavioral performance in the SIT (h), SPT (i), TST (j), and FST (k) for the indicated groups (Exp \#2). K-Means clustering of individuals in the SIT x SPT x [TST x FST] matrix

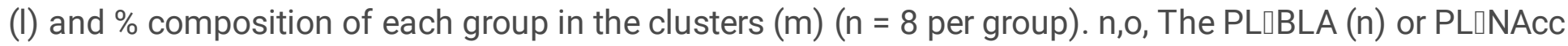
(o) circuits, which were labeled with $\mathrm{hM} 4 \mathrm{D}(\mathrm{Gi})$ by injection of viral vectors (a). $\mathrm{p}-\mathrm{s}$, S5-induced c-Fos expression levels in the BLA ( $n=3-4$ per group) ( $p$ and $q)$ and NAcc $(n=3-4$ per group) ( $r$ and $s)$ after CNO treatment (Exp \#1). t, Basal CORT levels in the indicated groups on post-stress day 22 ( $n=10$ per group). $u-x$, Behavioral performance in the SIT (u), SPT (v), TST (w), and FST (x) for the indicated groups (Exp 
\#2). Data are mean \pm SEM. Gray circles represent individual data points. *, difference compared to control; \#, difference compared to CRST. *, \#, p< 0.05; **, \#\#, p < 0.01 (Student's t-test; One-way ANOVA followed by Newman-Keuls post-hoc test). See Supplementary Table 4 for statistical details.

a

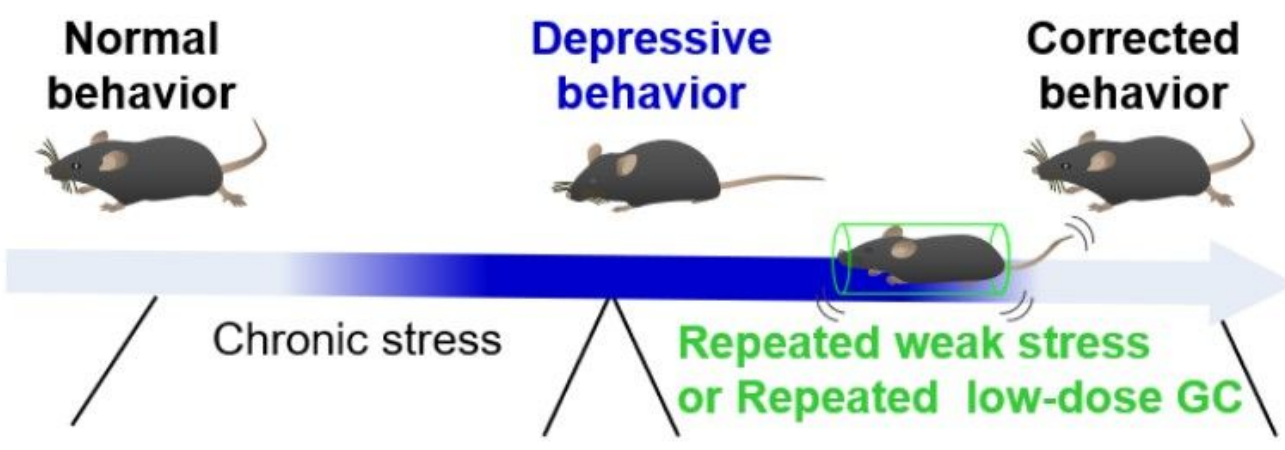

b

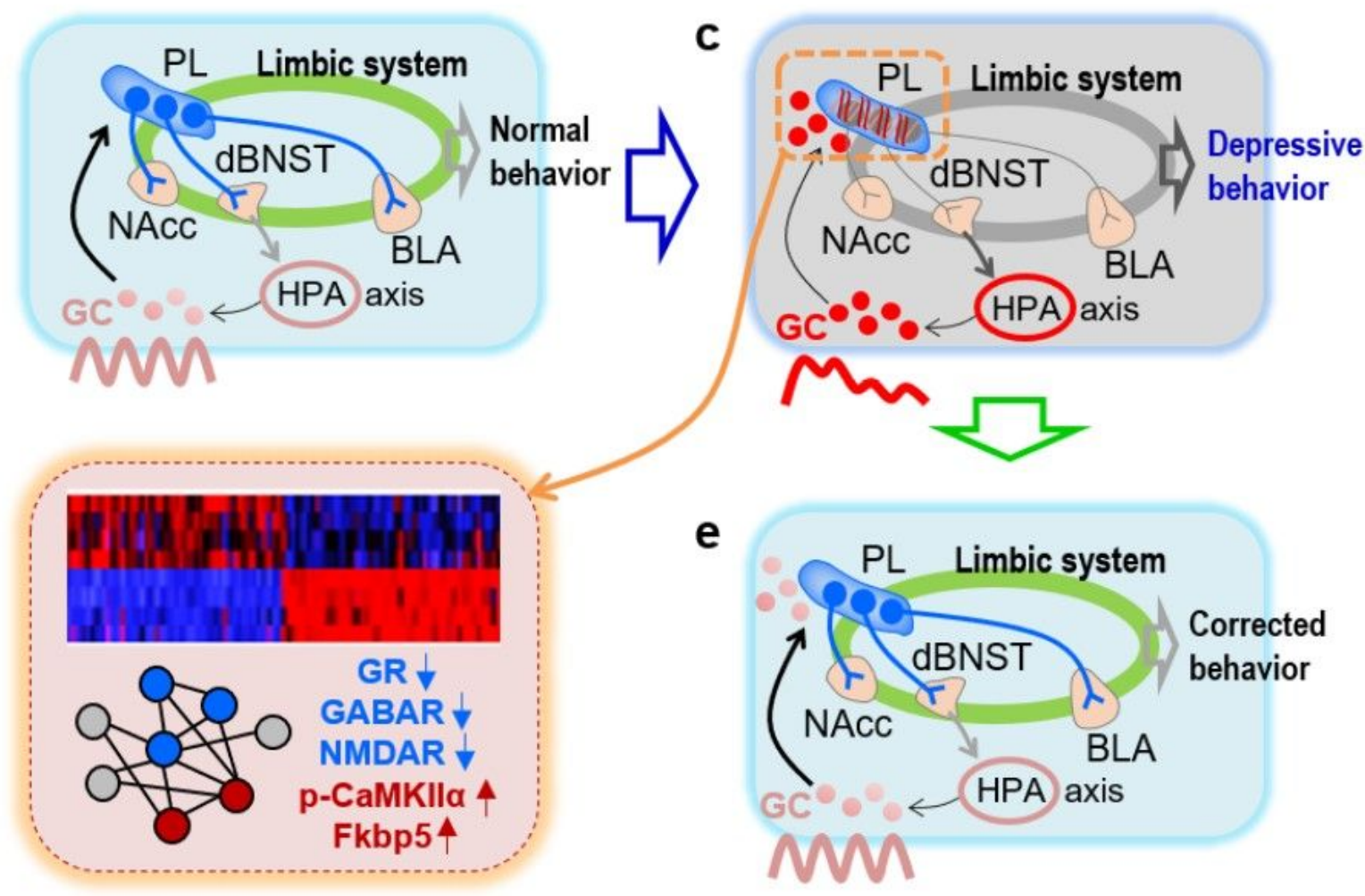

Figure 10

A summary and hypothetical model for the recovery of adaptively changed stress gains by treatment with strategic behavioral stress or GC. a, Chronic stress produces adaptive changes in the brain, and cumulative effects of chronic stress cause persistent depressive behavior. Repeated treatment with a short sequence of behavioral stress or GC in mice subjected to chronic stress reverses the stress-induced adaptive changes and rescues depressive behavior. $b$, The schematic presentation of the brain with

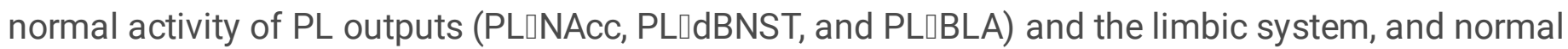
activity of the HPA axis including basal GC release at a normal circadian cycle and its feedforward effects on the PL. c,d, Chronic stress produces the PL overstimulated primarily due to increased GC (c), which results in genome-wide gene expression alteration (d). The reversal of the stress-induced changes 


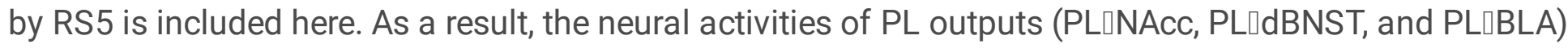
and the limbic system are downregulated or altered, and the basal GC release is enhanced and trailed off from a normal circadian cycle (c). The expression levels of GR, GABAR, NMDAR, p-CaMKIla and Fkbp5 in the $\mathrm{PL}$ are changed by chronic stress, and their physiological effects on depressive behaviors are characterized in the present study (d). e, Repeated treatment with behavioral stress or GC reverses the stress-induced altered neural activities of PL outputs, the altered gene expression profiles, the altered activity of the HPA axis including basal GC release, and the altered feedforward GC effects on the PL.

\section{Supplementary Files}

This is a list of supplementary files associated with this preprint. Click to download.

- SupplementaryTable1cFosmappingNC.docx

- SupplementaryTable2.GeneprofileofthePLNC.docx

- SupplementaryTable3.GeneprofileofthePLNC.docx

- SupplementaryTable4.StatisticalAnalysisNC.pdf

- sFIGURES.docx 